\title{
The Taxus genome provides insights into paclitaxel biosynthesis
}

\author{
Xingyao Xiong ${ }^{1,2,9}$, Junbo Gou ${ }^{2,9}$, Qinggang Liao ${ }^{2,9}$, Yanlin $\mathrm{Li}^{1,3,9}$, Qian Zhou ${ }^{2,4}$, Guiqi $\mathrm{Bi}^{2}$, Chong $\mathrm{Li}^{2}$,

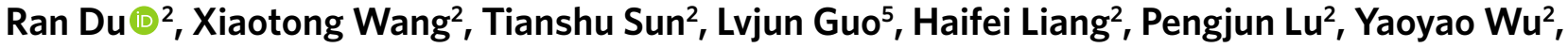 \\ Zhonghua Zhang $\oplus^{6}$, Dae-Kyun $\mathrm{Ro}^{2,7}$, Yi Shang $\oplus^{8}$, Sanwen Huang ${ }^{2}{ }^{2}$ and Jianbin Yan $\oplus^{2} \bowtie$
}

\begin{abstract}
The ancient gymnosperm genus Taxus is the exclusive source of the anticancer drug paclitaxel, yet no reference genome sequences are available for comprehensively elucidating the paclitaxel biosynthesis pathway. We have completed a chromosome-level genome of Taxus chinensis var. mairei with a total length of 10.23 gigabases. Taxus shared an ancestral whole-genome duplication with the coniferophyte lineage and underwent distinct transposon evolution. We discovered a unique physical and functional grouping of CYP725As (cytochrome P450) in the Taxus genome for paclitaxel biosynthesis. We also identified a gene cluster for taxadiene biosynthesis, which was formed mainly by gene duplications. This study will facilitate the elucidation of paclitaxel biosynthesis and unleash the biotechnological potential of Taxus.
\end{abstract}

T axaceae, a widespread family of non-flowering conifers with substantial economic value, contains six extant genera and over 28 species $^{1}$. Taxus is the largest genus in Taxaceae, including common species such as T. chinensis, T. brevifolia and T. baccata, and it is mainly distributed in Asia, North America and Europe ${ }^{2}$. For decades, Taxus has served as a natural source of paclitaxel (trade name Taxol), a well-known chemotherapy agent against various cancers $^{3}$. But plant-derived paclitaxel suffers from a short supply due to its low abundance in Taxus, limiting its clinical application. Multiple strategies have been employed to address supply issues ${ }^{4}$, and promising progress has been made in $\mathrm{chemical}^{5}$ and semichemical synthesis ${ }^{6}$, direct extraction from Taxus cell lines ${ }^{7}$, fermentation of endophytic paclitaxel-producing fungi ${ }^{8}$ and metabolic engineering of paclitaxel production using heterologous systems ${ }^{9}$.

As a tetracyclic diterpene, paclitaxel is biosynthesized by a complex metabolic pathway ${ }^{10}$. The paclitaxel pathway starts with geranylgeranyl diphosphate (GGPP) synthesis through the condensation of isoprenyl diphosphate and dimethylallyl diphosphate ${ }^{11}$. GGPP is then cyclized by taxadiene synthetase (TS), generating a unique diterpene skeleton, taxadiene ${ }^{12}$. Taxadiene is subsequently decorated by a series of reactions including hydroxylation, oxidation, epoxidation, acylation and benzoylation to generate the final product via catalysis by various enzymes (for example, hydroxylase, oxidase, epoxidase, oxomutase and transferase $)^{13-15}$. To date, over 20 enzymes have been identified in the paclitaxel biosynthetic pathway. However, several essential steps in the pathway, such as $\mathrm{C} 1$ hydroxylation, C9 oxygenation and oxetane formation, remain to be clarified. Moreover, studies have shown that jasmonates, gibberellin, auxin and ethylene are involved in the regulation of paclitaxel biosynthesis to maintain a delicate balance between growth and defence in Taxus $^{16-18}$. Several transcription factors (TFs), including
AP2/ERF, WRKY, MYC and MYB, have been found to regulate the expression of paclitaxel biosynthetic genes ${ }^{19-21}$. However, the comprehensive regulatory mechanisms underlying the growth-defence trade-off are still poorly understood.

A complete Taxus genome sequence can provide valuable bioinformatic and genetic resources to understand paclitaxel biosynthesis and regulatory mechanisms in depth, but the size and complexity of the Taxus genome (2C-value, 22.3-24.3 picograms) have hindered its de novo draft genome assembly to date ${ }^{22}$. Here, we have successfully assembled the Taxus genome, and we present a reference-grade genome sequence of $T$. chinensis var. mairei containing 10.23 gigabases $(\mathrm{Gb})$ of data with contig N50 of 2.44 megabases (Mb), $9.86 \mathrm{~Gb}$ of which was assigned to 12 pseudochromosomes. We demonstrate that the CYP725A (cytochrome P450) genes, closely related to paclitaxel biosynthesis, have evolved independently in a unique physical and functional grouping in the Taxus genome. Moreover, we have uncovered a gene cluster for taxadiene biosynthesis that contains a new type of TS. These results contribute to our understanding of the biological and evolutionary questions regarding paclitaxel biosynthesis and provide insights into the genome structure and organization of gymnosperms.

\section{Results}

Taxus genome sequencing, assembly and annotation. To build a chromosome-level genome assembly of Taxus, genomic DNA was extracted from endosperm calli. The endosperm of $T$. chinensis var. mairei seeds with haploid chromosomes was used to culture the callus, as it could prevent the influence of heterozygous elements in the genome assembly. $K$-mer analysis showed that the genome size of $T$. chinensis var. mairei was approximately $10 \mathrm{~Gb}$ (Extended Data Fig. 1a), which is consistent with the results

'College of Horticulture, Hunan Agricultural University, Changsha, China. ${ }^{2}$ Shenzhen Branch, Guangdong Laboratory for Lingnan Modern Agriculture, Shenzhen Key Laboratory of Agricultural Synthetic Biology, Genome Analysis Laboratory of the Ministry of Agriculture and Rural Affairs, Agricultural Genomics Institute at Shenzhen, Chinese Academy of Agricultural Sciences, Shenzhen, China. ${ }^{3}$ Engineering Research Center for Horticultural Crop Germplasm Creation and New Variety Breeding, Ministry of Education, Changsha, China. ${ }^{4}$ Peng Cheng Laboratory Artificial Intelligence Research Center No. 2, Shenzhen, China. ${ }^{5}$ MOE Key Laboratory of Bioinformatics, Tsinghua-Peking Joint Center for Life Sciences, School of Life Sciences, Tsinghua University, Beijing, China. ${ }^{6}$ College of Horticulture, Qingdao Agricultural University, Qingdao, China. ${ }^{7}$ Department of Biological Sciences, University of Calgary, Calgary, Alberta, Canada. ${ }^{8}$ The AGISCAAS-YNNU Joint Academy of Potato Sciences, Yunnan Normal University, Kunming, China. ${ }^{9}$ These authors contributed equally: Xingyao Xiong, Junbo Gou, Qinggang Liao, Yanlin Li. 凶e-mail: huangsanwen@caas.cn; jianbinlab@caas.cn 
from the flow cytometry tests ${ }^{23}$. A de novo assembly of the Taxus genome was achieved by PacBio continuous long reads $(318.05 \mathrm{~Gb})$ and augmented with Illumina whole-genome sequencing reads $(693.73 \mathrm{~Gb})$ (Supplementary Table 1). After the application of high-throughput/resolution chromosome conformation capture (Hi-C) (Supplementary Table 2), 9.86 Gb of sequence data could be assigned to 12 pseudochromosomes (Extended Data Fig. 1b and Supplementary Table 3), which covered $96.28 \%$ of the genome (Supplementary Table 4). We finally obtained the genome sequence with a total length of $10.23 \mathrm{~Gb}$ and a contig N50 of $2.44 \mathrm{Mb}$ (Fig. 1a and Supplementary Table 4).

On the basis of the genomic information, 42,746 protein-coding genes were further annotated by integrating transcriptome data, homologous alignments and ab initio gene models. In total, $73.02 \%$ of the genes $(31,214$ out of 42,746$)$ could be supported by RNA-seq data (Extended Data Fig. 1c and Supplementary Table 5). The BUSCO analysis further demonstrated that 1,052 of the 1,614 core genes were complete, showing relatively high completeness of the assembled genome in gymnosperms (Supplementary Table 6). Furthermore, 36,518 coding genes, accounting for $85.43 \%$ of the total predicted genes, were assigned to functional categories with an $E$-value less than $10^{-5}$ (Supplementary Table 7).

Taxus experienced a whole-genome duplication event in the cupressophyte clade. Given that whole-genome duplication (WGD) is a important evolutionary force contributing to the expansion of plant genome $\operatorname{size}^{24}$, we investigated whether Taxus had experienced any WGD events. We built a paralogous gene pair set by performing an all-against-all blastp search. The number of synonymous substitutions per synonymous site $\left(K_{\mathrm{s}}\right)$ of paralogues was calculated using the gene pair set. As shown in Fig. 1b, the frequency of $K_{\mathrm{s}}$ values exhibited an apparent decay without a natural distribution with increasing $K_{\mathrm{s}}$ values, which indicated that no recent WGD event occurred in the Taxus genome. Moreover, we noticed that most of the $K_{\mathrm{s}}$ values were less than 0.8 , indicating that gene duplication in combination with saturation and stochasticity effects may obscure $\mathrm{WGD}^{24}$. We further used MCScanX to produce 8,148 syntenic gene pairs from the all-against-all blastp data and entered them into the $K_{\mathrm{s}}$ and distance-transversion rate at fourfold degenerate sites (4DTv) calculations. The results showed two signature peaks located at 2.1 for $K_{s}$ (Fig. 1b) and 0.7 for 4DTv (Extended Data Fig. 1d), suggesting the presence of an ancient WGD in Taxus. Together with previous studies that revealed an ancient WGD event (WGD- $\zeta$ ) in the common ancestor of angiosperms and gymnosperms ${ }^{24,25}$, all the above results suggest that Taxus shared the common ancient WGD with other coniferophyte lineages.

Taxus genome expansion is linked with retrotransposons. Except for the role of WGD in enlarging the Taxus genome size, we noticed that repetitive sequences constituted a important component of the Taxus genome (Supplementary Table 8). There was a total of $7.79 \mathrm{~Gb}$ of repetitive sequences, occupying $76.09 \%$ of the entire genome (Supplementary Table 8 ). Among these repetitive sequences, long terminal repeat (LTR) retrotransposons accounted for the highest proportion at $52.38 \%$ (Supplementary Table 8 ). The insertion time analysis revealed that LTR insertion was a continuous process, and approximately $40 \%$ of the insertions occurred 8 to 24 million years ago (Ma) (Fig. 1c). This feature of continuous insertion in the Taxus genome was distinctly different from that in the rice genome, where almost 95\% of LTR insertions occurred within the last 5 million years ${ }^{26}$. Considering that LTR insertion in Norway spruce and ginkgo mainly occurred 12-24 and 16-24 $\mathrm{Ma}^{23,25}$, the continuous insertion of LTRs might be a common phenomenon in gymnosperms.

To further explore the evolution of LTR in Taxus, we analysed the phylogenies of LTR retrotransposons in a few representative gymnosperm and angiosperm plants. Amino acid sequence similarities within the reverse transcriptase domain of the Ty3/ Gypsy retrotransposons (Gypsy) and Ty1/Copia retrotransposons (Copia) were used to construct phylogenetic trees. As shown in Fig. 1d, the Gypsy superfamily members of the gymnosperms ginkgo and Picea were distributed in families II-VII, while those of angiosperms mainly belonged to family VIII. In contrast, Taxus Gypsy elements not only were distributed in families II-VIII but also evolved a highly species-specific family (family I), suggesting the expansion of specific Gypsy elements after Taxus speciation. Similarly, the unique expansion phenomenon in Taxus was also observed in the phylogenies of the Copia superfamily (Fig. 1d). Moreover, family V consisted of only Taxus LTRs in the Copia phylogenetic tree displaying a Taxus-specific amplification burst. In addition, Taxus was distributed in family IV, where the gymnosperms ginkgo and Picea were located, and families I-III also contained angiosperms, suggesting that Taxus LTRs were placed in a unique position compared with other selected species. These results suggest that the Gypsy and Copia superfamilies of Taxus have undergone a relatively unique evolutionary pattern, especially the specific Gypsy family I and Copia family V.

Evolution of gene families and elevated secondary metabolism in Taxus. To understand the context of metabolic networks during Taxus evolution, we compared orthologous genes between Taxus and selected gymnosperms, angiosperms and cryptogams (Fig. 1e). In the 35,298 identified orthologous gene families (Supplementary Table 9), we found that 6,533 gene families were shared by the selected species, illustrating their evolutionary conservation (Fig. 1e). Compared with the selected species, 2,339 gene families were exclusive to Taxus (Fig. 1e). In addition, 1,378 gene families experienced loss, while 142 and 41 families underwent expansion and contraction in Taxus, respectively (Fig. 1f).

Taxus contains 9,747 unique genes (Fig. 1e and Supplementary Table 10), many of which are enriched in the biosynthesis of specialized metabolites, including terpenes, phenylpropanoids and flavones (Supplementary Table 11). For instance, 57 gene families were annotated to be cytochrome P450 (CYP450) gene families (Supplementary Table 10). Gene expansion analysis demonstrated that 979 genes were enriched in ADP binding, oxidoreductase activity, flavin adenine dinucleotide binding, transferase activity and signal transduction, among other functions (Extended Data Fig. 1e and Supplementary Table 12), with eight gene families being associated with CYP450 (Supplementary Table 13). Pfam functional analysis further showed that the Taxus genes were enriched in CYP450 gene families (PF00067.22, $P<0.01$ ) and TFs (PF13837.6, $P<0.01$; and PF00847.20, $P<0.01$ ) (Supplementary Table 14). KEGG analysis indicated that the gained and expanded gene families were enriched in a total of 36 and 41 KEGG pathways, respectively, including one phenylpropanoid (ko00940) and three terpenoid metabolic pathways (ko00900, ko00130 and ko00902) (Supplementary Tables 11 and 15).

Evolution and genomic organization of Taxus CYP450s. Given that CYP450s participate in almost half of the enzymatic reactions in paclitaxel biosynthesis ${ }^{27}$, we analysed Taxus CYP450 families and identified 649 CYP450 genes from the present genome using the reported HMM model (PF00067). These CYP450s can be divided into two catalogues: A-type and non-A-type. The A-type CYP450s included only the CYP71 clan, which consisted of 17 families and 325 genes (Extended Data Fig. 2a and Supplementary Table 16), while the non-A-type CYP450s contained 12 clans that were composed of 27 families and 324 genes (Extended Data Fig. 2b and Supplementary Table 16). Phylogenomic analyses showed that the CYP750 and CYP725 families were obviously expanded in Taxus compared with 68 other representative species, which covered Zygnematophyceae and Sapindaceae (Fig. 2a, 


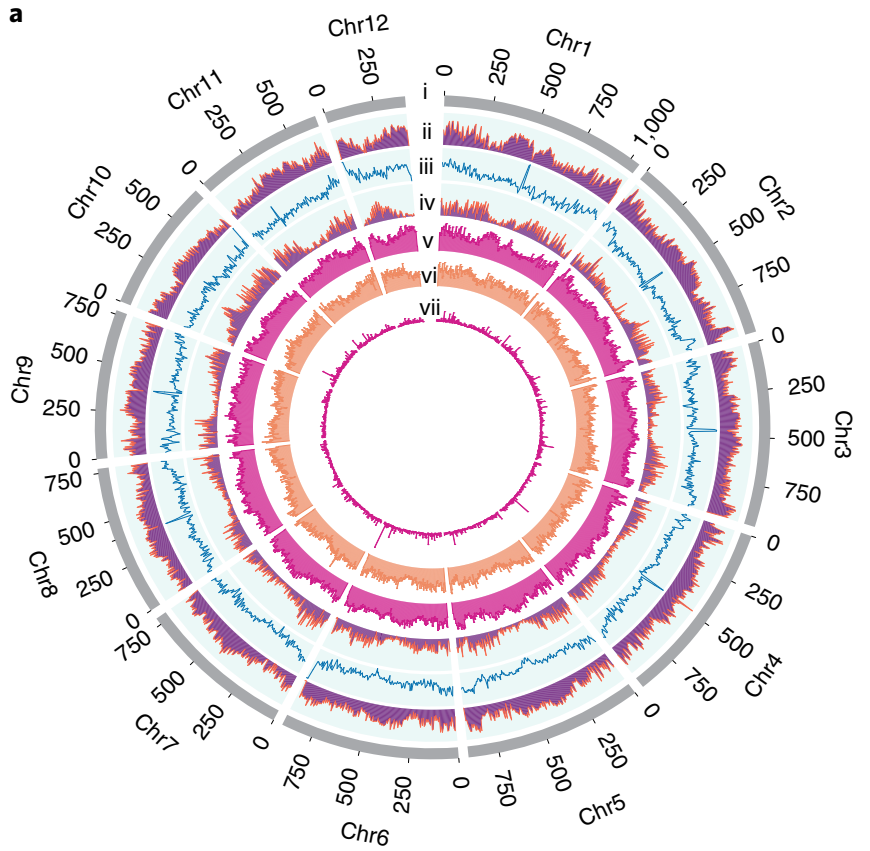

b
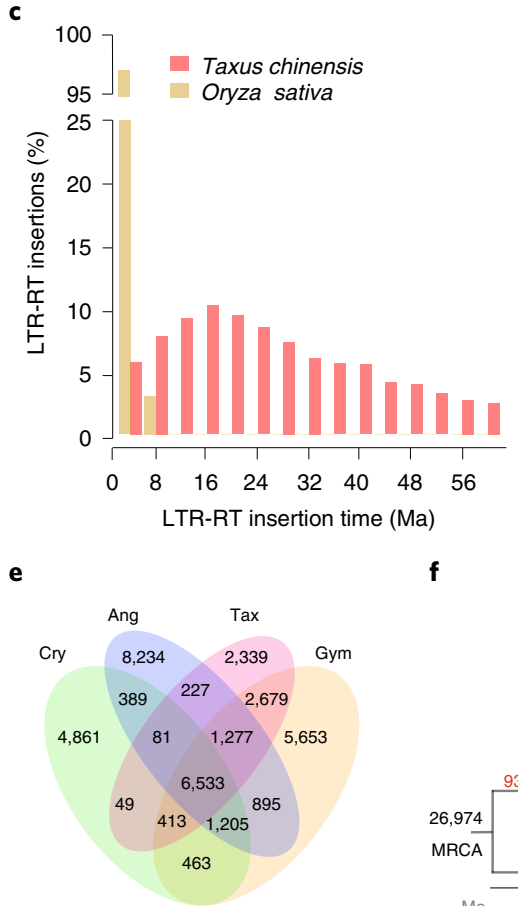

d

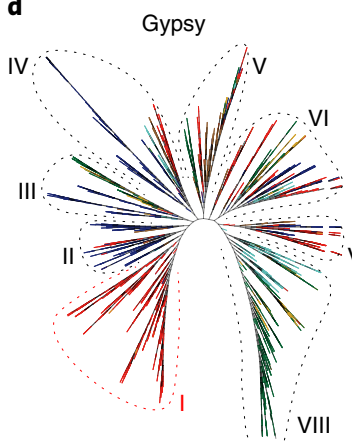

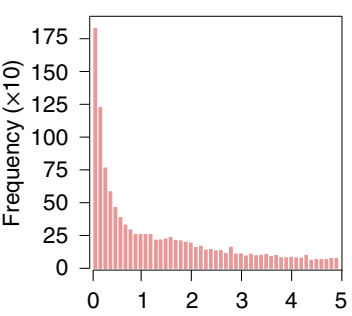

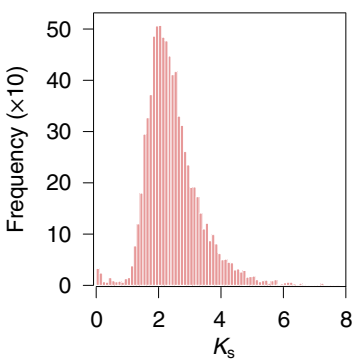

Copia
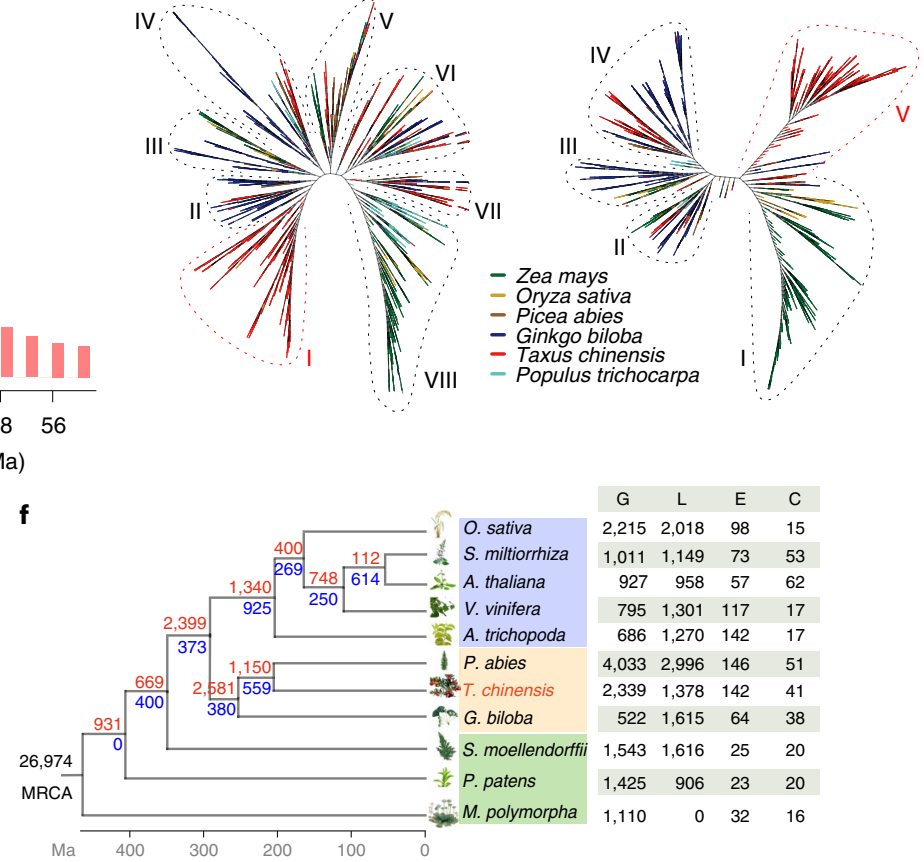

Fig. 1 | Genomic features of $\boldsymbol{T}$. chinensis var. mairei. a, Genomic landscape of the 12 assembled pseudochromosomes. Track i represents the length of the pseudochromosomes (Mb); ii-iv represent repeat element density, GC content and distribution of gene density, respectively; and v-vii show the distribution of Ty3/Gypsy, Ty1/Copia and unknown LTRs, respectively. These metrics are calculated in $5 \mathrm{Mb}$ windows. $\mathbf{b}$, WGD analysis based on the substitution rate distribution of paralogues. Top, histogram of the $K_{s}$ distribution from Taxus paralogues based on an all-to-all blast to total genes. Bottom, $K_{\mathrm{s}}$ distribution of paralogues based on syntenic analysis. The $K_{\mathrm{s}}$ values were calculated using the $\mathrm{YN}$ model in KaKs_calculator. c, Expansions and diverse sets of LTR elements in the Taxus genome. The histogram shows distributions of insertion times calculated for LTRs in Taxus and rice, using mutation rates (per base year) of $7.3 \times 10^{-10}$ for Taxus and $1.8 \times 10^{-8}$ for rice. The LTR-retrotransposon (LTR-RT) insertions of T. chinensis var. mairei and Oryza sativa are shown as columns in different colours. $\mathbf{d}$. Heuristic maximum likelihood trees of Ty3/Gypsy (shown as Gypsy) and Ty1/Copia (shown as Copia) from six plant species. The two trees were constructed from amino acid sequence similarities within the reverse transcriptase domains of Gypsy and Copia from six plant species. Gypsy elements are divided into eight families (I-VIII), and Copia contains five families (I-V). The representative plants are shown as coloured lines. e, Venn diagram for orthologous protein-coding gene clusters in cryptogam (Cry), angiosperm (Ang), gymnosperm (Gym) and T. chinensis var. mairei (Tax). The cryptogams include M. polymorpha, Physcomitrella patens subsp. patens and Selaginella moellendorffii. The angiosperms include Amborella trichopoda, V. vinifera, Arabidopsis thaliana, Salvia miltiorrhiza and O. sativa. The gymnosperms include Picea abies and Ginkgo biloba. The number in each sector of the diagram represents the total number of genes across the four comparisons. f, Evolution analysis of gene families in Taxus and selected plants. The red numbers on the branches of the phylogenetic tree indicate the number of expanded gene families, and the blue numbers refer to the number of constricted gene families. The supposed most recent common ancestor (MRCA) contains 26,974 gene families. G, L, E and C in the table at right represent the number of gains, losses, expansions and constrictions in the gene families among 11 plant species. 
a

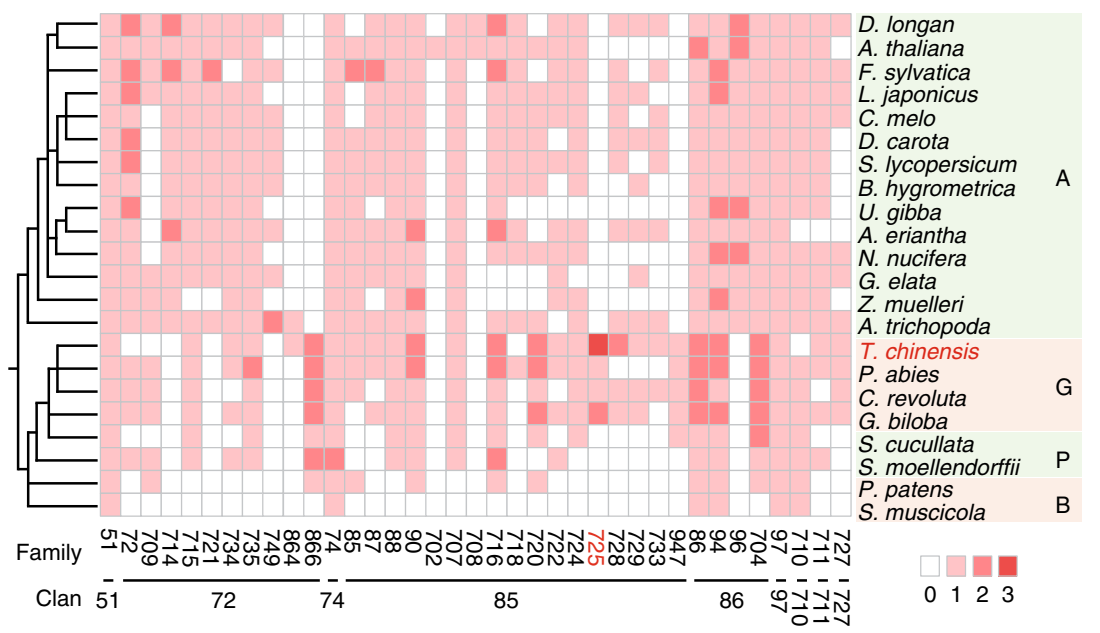

b

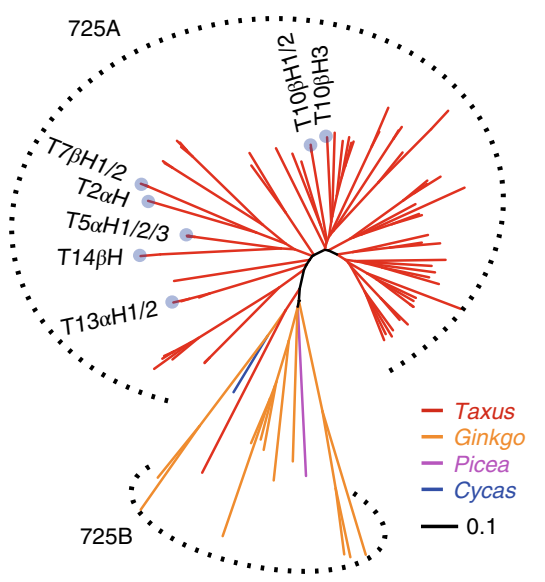

c

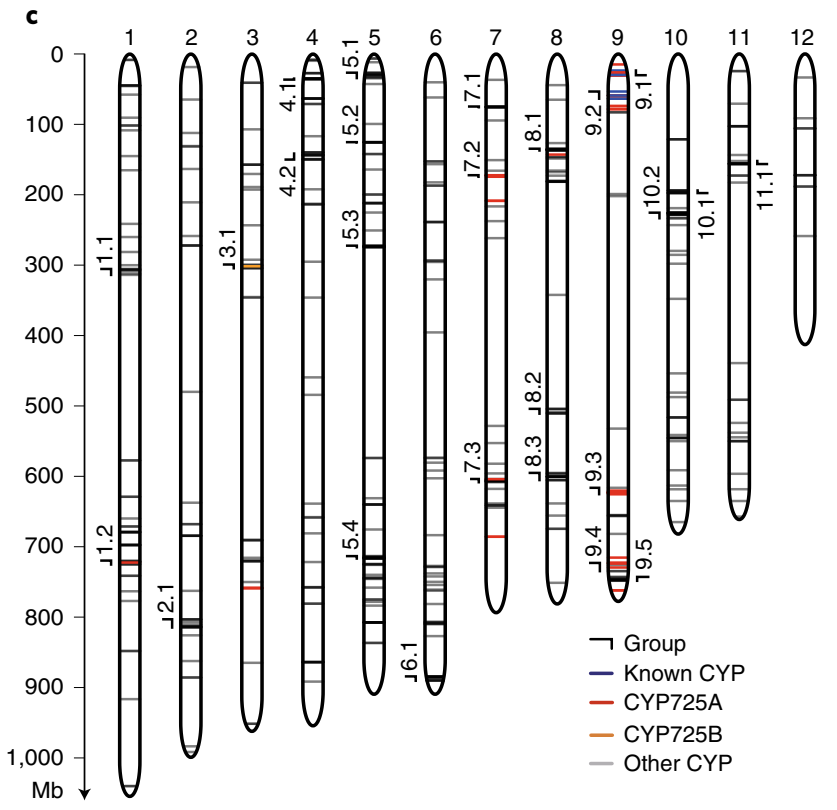

d

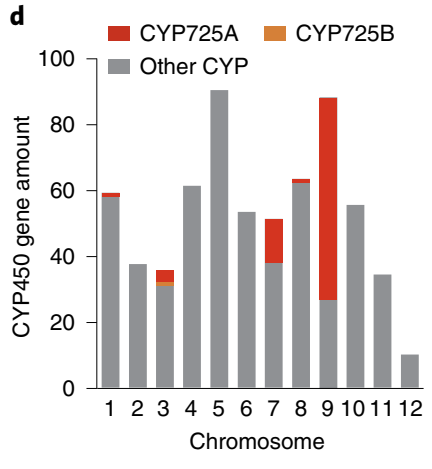

e

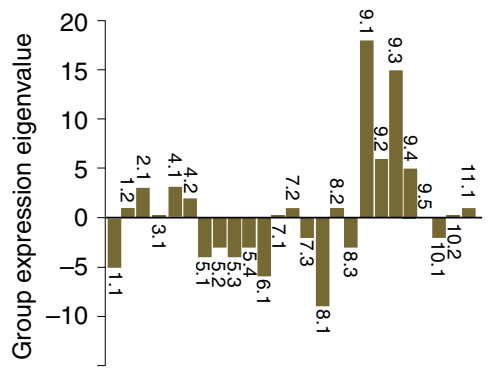

f

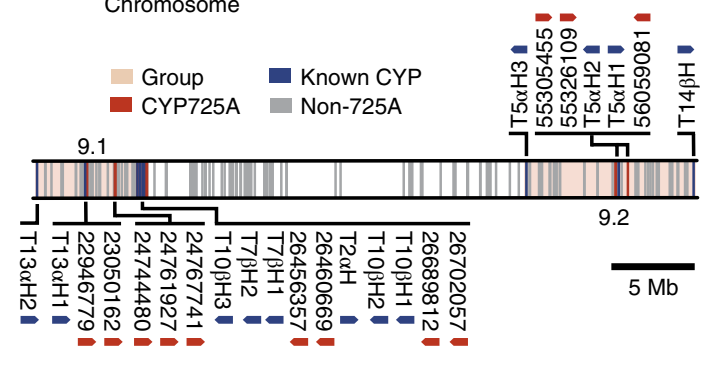

Fig. 2 | Evolution and genomic architecture of Taxus CYP450s. a, Phylogenomic analysis of the non-A-type CYP450s in the representative plant species. A, angiosperms; G, gymnosperms; P, pteridophytes; B, bryophytes. The colour of each block is based on the number of genes in each family, and $0,1,2$ and 3 indicate that this number ranges from 0, 1-10, 10-50 and 50-100 genes, respectively. b. Phylogenetic analysis of the CYP725 subfamily in T. chinensis var. mairei (Taxus), Ginkgo biloba (Ginkgo), Picea abies (Picea) and C. revolute (Cycas). The dotted outline shows the gene spheres of the CYP725A and CYP725B subfamilies. The light blue dots on the ends of the phylogenetic branches represent the known paclitaxel pathway CYP725A genes and their homologues. The neighbour-joining tree was constructed by Interactive Tree Of Life (iTOL) software. The evolutionary distances were analysed by the $p$-distance method, and the branch lengths were scaled by the bar. c, Distribution of CYP450 genes on the 12 pseudochromosomes in Taxus. Each short line on the pseudochromosomes represents a CYP450 gene. CYP725As, CYP725Bs and the other CYP450s are marked by red, orange and grey lines, respectively. The known CYP450s in the paclitaxel biosynthesis pathway (known CYP) are shown in blue. The CYP450 groups ( $\geq 7$ CYP450 genes and $\leq 5.26 \mathrm{Mb}$ of gene spacing between two adjacent CYP450s) are labelled outside of the corresponding positions on the pseudochromosomes. $\mathbf{d}$, Histogram of the number of CYP450 genes on each pseudochromosome. The CYP725 genes (shown in red and orange) were mainly distributed on pseudochromosome 9, while the other CYPs (shown in grey) were distributed randomly on 12 pseudochromosomes. The $y$ axis represents the number of CYP450 genes. e, Group-based gene expression profiles in response to methyl jasmonate (MeJA) treatment. RNA sequencing analysis was performed with the low-paclitaxel-yielding cell line (LC) treated with $100 \mu \mathrm{M}$ MeJA for $4 \mathrm{~h}$. The expression of the gene group was calculated by the sum of the expression levels of each CYP450, and each upregulated and downregulated CYP450 was calculated as 1 and -1 , respectively, on the basis of their reads per kilobase per million reads values. $\mathbf{f}$, Map of CYP725As located in groups 9.1 and 9.2. The ranges of the gene groups on pseudochromosome 9 are marked in pink. CYP725As and the other genes are marked by red and grey vertical lines, respectively. The known CYP450s in the paclitaxel biosynthesis pathway (known CYP) are shown in blue. The arrows show gene orientations.

Extended Data Fig. 3a,b and Supplementary Table 17). The CYP750 family was reported to participate in the biosynthesis of thujone monoterpene, which is involved in defence responses (for example, resistance against herbivore feeding $)^{28}$, while CYP725 genes were known to contribute to paclitaxel biosynthesis ${ }^{29}$. Phylogenetic analysis of these CYP725 genes further showed that they could be categorized into the CYP725A and CYP725B subfamilies (Fig. 2b). The CYP725A subfamily (a total of 79 genes) exhibited specificity 
to Taxus, whereas the CYP725B subfamily was universal in gymnosperm plants (including Picea, Cycas, Ginkgo and Taxus) (Fig. 2b), which suggested that CYP725A underwent independent evolution in Taxus. Considering that all the previously defined CYP450 genes in the paclitaxel pathway belong to the CYP725A subfamily, these results suggest that the expansion of the CYP725A subfamily played vital roles in the evolution of paclitaxel biosynthesis in Taxus.

We noticed that most CYP725A genes (74.68\%) were located on pseudochromosome 9 (Fig. 2c,d), exhibiting a distinct non-uniform distribution. Gene location analysis further revealed that the Taxus CYP450 genes were not distributed randomly but tended to organize into different gene groups, 25 of which were detected in the genome (Fig. 2c). We found that nearly all these groups, except groups 1.2 and 5.1, contained gene members from no more than three CYP450 families, and 11 groups had only one CYP450 family (Supplementary Table 18), suggesting that the grouping of CYP450 genes on the genome had an obvious family aggregation pattern. Furthermore, as an essential phytohormone in the biosynthesis of secondary metabolites ${ }^{30}$, jasmonate is closely related to the expression regulation of CYP450 genes in Taxus (Fig. 2e). Under jasmonate treatment, eight groups showed an obviously increased expression level, and ten groups showed clear inhibition of gene expression (Fig. 2e, Extended Data Fig. 4 and Supplementary Table 18). These results suggest that the CYP450s in the majority of groups were coexpressed under jasmonate treatment, implying that the grouping of CYP450 genes had some coordination of physiological functions.

The gene expression levels of four gene groups (group 9.1-9.4) on pseudochromosome 9 were upregulated most prominently in the presence of jasmonate (Fig. 2e). More interestingly, groups 9.1 and 9.2 contained all known CYP725A subfamily genes related to paclitaxel biosynthesis and 12 undefined CYP725As (Fig. 2f). The expression profiles of these two groups of CYP725A genes showed that $88 \%$ of CYP725As were highly expressed in roots, $79 \%$ of CYP725As were highly expressed in the high-paclitaxel-yielding cell line (HC) and $88 \%$ of CYP725As were upregulated after jasmonate treatment (Supplementary Table 19), which is consistent with the results on the increased level of baccatin III and paclitaxel in the Taxus cell line under jasmonate treatment (Supplementary Fig. 1). These results suggest that the two groups are likely to contain most of the paclitaxel pathway genes that arose during Taxus evolution.

Taxadiene biosynthetic genes are arranged in gene clusters. PlantiSMASH $^{31}$ analysis further showed that a potential gene cluster related to terpene biosynthesis was presented in group 9.2 (Fig. 3a and Supplementary Table 20). The gene cluster contained two TS genes (TS2 and TS3, sharing $99.96 \%$ nucleotide sequence identity), two $\mathrm{T} 5 \alpha \mathrm{H}$ genes ( $\mathrm{T} 5 \alpha \mathrm{H} 1$ and $\mathrm{T} 5 \alpha \mathrm{H} 2$, sharing $98.67 \%$ nucleotide sequence identity) and two unknown CYP725As (Fig. 3a, Supplementary Table 21 and Supplementary Figs. 2 and 3). Moreover, the genes in the cluster showed a highly coordinated tissue expression pattern and expression consistency in response to jasmonate treatment (Fig. 3a), suggesting that the genes could be functionally related. TS2 and TS3 were located adjacent to T5 $\alpha \mathrm{H} 1$ and T5 $\alpha \mathrm{H} 2$ (Fig. 3a), suggesting that the genes involved in the first two paclitaxel biosynthetic steps were organized by a tandem gene duplication event during Taxus genomic evolution. The $K_{\mathrm{s}}$ value of these duplicated genes suggested that this $T S-T 5 \alpha H$ duplication occurred approximately $1.15 \mathrm{Ma}$. In addition to the TS and T5 $\alpha H$ genes assembled in the cluster, additional TS (TS1) and $T 5 \alpha H(T 5 \alpha H 3)$ genes are located downstream and upstream of the cluster, respectively (Fig. 3a). Biochemical assays further confirmed that TS $1 / 2$ and $T 5 \alpha H 1 / 2 / 3$ have TS activity (Fig. $3 b$ ) and taxa-4(5),11(12)-diene-5 $\alpha$-hydroxylase activity (Fig. 3c and Supplementary Fig. 4), respectively, demonstrating that the copied genes possessed the corresponding enzyme activities in T. chinensis var. mairei.
We further studied the kinetic properties of TS1 and TS2. The $K_{\mathrm{m}}$ value of TS2 was approximately 1.5 times higher than that of TS1, but the turnover number $\left(k_{\text {cat }}\right)$ of TS2 was nearly 2 times greater than that of TS1, indicating that TS2 might have a higher catalytic efficiency than TS1 (Fig. 3d). Moreover, exogenous jasmonate treatment resulted in an obviously higher level of TS2 than TS1 transcripts (Fig. 3e), implying that TS2 could play a role in paclitaxel biosynthesis in response to different environmental and developmental cues via jasmonate signalling. Sequence identity analysis showed that TS2 shared only $77-78 \%$ protein sequence identity with TS1 and T. brevifolia TS (TbTS), which is much lower than the sequence similarity (over 90\%) within the previously reported TS genes (Supplementary Table 21), suggesting that TS2 is a unique TS gene that diverged from TS1 and TbTS. Phylogenetic tree analysis further confirmed that a Taxus-specific gene duplication event approximately 33.2 Ma resulted in two distinct types of TS genes (Extended Data Fig. 5), demonstrating that TSs were encoded by two types of TS genes resulting from gene duplication events in Taxus. Together, these results suggest that the genes involved in the two initial steps of the paclitaxel biosynthesis pathway are arranged in a gene cluster named the 'taxadiene gene cluster'. The taxadiene gene cluster might be formed by gene duplications and neofunctionalization in Taxus and may be somewhat similar to previous studies on operon-like gene clusters in plants ${ }^{32,33}$.

Furthermore, we established a gene-to-gene coregulation network using three rounds of subtraction screening with RNA-seq datasets. The network could cover all known paclitaxel biosynthetic genes (Extended Data Fig. 6 and Supplementary Table 22), indicating its comprehensiveness and high credibility. We identified 17 CYP725A genes, 3 transferases and 10 TFs with this network, which was strongly associated with known paclitaxel biosynthetic genes (Supplementary Tables 23 and 24). Real-time quantitative PCR assays confirmed that the expression of certain genes could be induced by jasmonates (Extended Data Fig. 6), implying that their encoded proteins could be investigated as potential enzymes in paclitaxel biosynthesis. Together, these results outline the biosynthesis pathway of paclitaxel in T. chinensis var. mairei (Fig. 3f) and provide valuable genetic resources for improving paclitaxel production through genetic breeding and synthetic biology.

\section{Discussion}

The absence of a chromosome-level genome sequence from Taxus has prevented in-depth phylogenomic studies of Taxus. Our study provides an example assembly of a complex genome in trees using various sequencing technologies on DNA from endosperm calli containing haploid chromosomes. Flow cytometry analysis indicated that the nuclear genome $(2 n)$ size of the diploid cells of Taxus was approximately $20.80-24.85 \mathrm{~Gb}$, nearly twice the haploid genome size evaluated by $k$-mer analysis (Extended Data Fig. 1a). The vast majority of HiFi sequences from Taxus leaves (diploid) could be mapped to the haploid genome (up to $95 \%$ ). Moreover, $75.81 \%$ of the $228,762,501$ single nucleotide polymorphisms, $44.97 \%$ of the 847,935 insertions and deletions, and $85.64 \%$ of the 64,927 structural variants were heterozygous. Taken together with the low heterozygosity $(0.02 \%)$ of $T$. chinensis var. mairei, these results demonstrate that the haploid genome assembly could basically represent its diploid genome, showing the advantages of using endosperm calli for genome assembly.

We found that the complete BUSCOs increased only from $64.7 \%$ to $65.2 \%$ when the N50 value was increased from 637 kilobases to $2.44 \mathrm{Mb}$ during the genome assembly. The low BUSCO value might be due to the limitations of the BUSCO reference dataset. The latest dataset version is embryophyta_odb10 (10 September 2020), containing 1,614 genes from single-copy genes of 50 species, including two bryophytes (Physcomitrella patens and Marchantia polymorpha), 
one fern (Selaginella moellendorffii) and 47 seed plants (all are angiosperms) but not including any genes of gymnosperms. Consistently, across all of the reported gymnosperm genomes, except for Gnetum montanum, the BUSCO values were not higher than $73 \%$, and four of these genomes had values lower than 51\% (Supplementary Table 6). The BUSCO value of the Taxus genome was $65.2 \%$, similar to that of Ginkgo biloba (69.4\%) and Pseudotsuga menziesii (67.8\%). To assess the Taxus genome quality more comprehensively, we mapped the Illumina DNA sequencing data $(\sim 693 \mathrm{~Gb})$ for the genome survey onto the assembled genome and found that up to $99.60 \%$ of the sequencing data could be mapped, indicating the integrity of the genome assembly. Moreover, we collected transcriptome data from Taxus organs, comprehensively covering eight tissues and cell lines (root, stem, leaf, bark, male strobili, female strobilus, HC and LC), and mapped the sequencing data to the Taxus genome. The results showed that the average overall mapping rate of transcriptome data to the genome reached $90.45 \%$ (Supplementary Table 25), suggesting the integrity of functional genes in the genome.

The Taxus genome contains $4.08 \mathrm{~Gb}$ of LTR retrotransposons, including $87.28 \%$ Gypsy and $12.35 \%$ Copia retrotransposons and a small proportion of unknown LTRs (0.37\%) (Supplementary Table 8). The LTR distribution analysis showed that LTRs tended to be distributed throughout the entire chromosome (Extended Data Fig. 7a). In particular, Copia tends to be enriched at the two ends of the chromosomes, while Gypsy is more enriched at the chromosome ends and central areas. Compared with previous studies in groundnuts ${ }^{34}$, the Taxus genome exhibited obvious differences in LTR distribution. The LTR retrotransposons of the groundnut genome are mainly distributed in the central regions of the chromosomes, close to the centromeres. This difference may come from the large disparity in genome size and the difference between angiosperms and gymnosperms.

The LTR insertions in the Taxus genome mainly occurred 8 to $24 \mathrm{Ma}$ during the long insertion period (4-60 Ma) (Fig.1c and Extended Data Fig. 7b-d), while the primary insertion times of LTRs in spruce and ginkgo were $12-24$ and $16-24 \mathrm{Ma}$ within their insertion span from 4 to $64 \mathrm{Ma}^{25,35}$. These results suggest that the Taxus genome has a similar LTR insertion time trend to that in the spruce and ginkgo genomes. The very long insertion time phenomenon might be related to the evolutionary characteristics of gymnosperms. It is generally accepted that gymnosperms are slow-evolving plants. Their morphology is highly conserved, which is supported by the high similarity between extant species and fossil records. Previous studies have shown that angiosperms and gymnosperms differ considerably in their mutation rates of molecular evolution per unit time, with gymnosperm rates being, on average, seven times lower than those of angiosperm species ${ }^{36}$. For this reason, an insertion time longer than 60 million years is common in gymnosperm genomes because of the much lower mutation rate. For example, up to $8.27 \%$ of LTRs were inserted into the ginkgo genome over 60 million years, and $13.31 \%$ of LTRs were inserted into the spruce genome over 60 million years ${ }^{25,35}$.

In addition to CYP450 enzymes, acetyltransferases play an essential role in paclitaxel biosynthesis, especially BAHD acyltransferases. We found 127 BAHD acyltransferases by identifying their conserved motifs (HXXXD and DFGWG). The BAHD acyltransferases in Taxus were mainly distributed in Clades I, II, VI and V. Clade V can be divided into three groups (Groups I-III), among which Group I contains all known BAHD acyltransferases in the paclitaxel biosynthesis pathway (Supplementary Fig. 5). It would be worthwhile to investigate whether Group I contains other acyltransferases that function in paclitaxel biosynthesis in the future (Supplementary Table 26). PlantiSMASH analysis indicated that the acyltransferase genes are not organized into any gene clusters. Genomic location analysis showed that the BAHD acyltransferase genes in paclitaxel biosynthesis were mainly distributed on chromosomes 1 and 9 (Extended Data Fig. 6b). Furthermore, TAT2 was colocalized with CYP450s in gene group 9.2 (Fig. 2c and Extended Data Fig. 6b). The relationship between CYP725As and acetyltransferases in paclitaxel evolution is an interesting aspect to study in the future.

In the Taxus genome, a total of 34 potential gene clusters related to secondary metabolism were found, including 13 saccharides, 7 terpenes, 1 alkaloid, 1 saccharide-terpene, 1 saccharide-polyketide,

Fig. 3 | Functional identification of the paclitaxel biosynthesis gene cluster. a, Genomic architecture and expression pattern of the taxadiene cluster. The arrows indicate the relative positions and directions of the genes in the cluster. Here, 55305455 and 55566094 indicate the starting and ending positions of the cluster on pseudochromosome 9. The two unknown CYP725A genes are represented by their gene starting positions (55326109 and 55305455). TS1 and T5 $\alpha \mathrm{H} 3$ are located at 72105619-72109598 and $49866845-49868629$ bp on chromosome 9 , respectively. The relative expression levels of taxadiene cluster genes in Taxus are based on their reads per kilobase per million reads values. The expression levels of genes with high sequence similarity were distinguished on the basis of sequencing read counts of the exons that include different bases, and adjusting the alignment threshold to no mismatch. RNA-seq datasets are from roots, leaves and bark of male plants (shown in green); two T. chinensis var. mairei half-sib cell lines, HC and LC (shown in yellow); and MeJA-treated LC (+MJ) and MeJA-untreated LC ( - MJ) (shown in orange). The data are shown as means \pm s.d. ( $n=3$ biologically independent samples). b. Analysis of TS activity in vitro. The purified recombinant TS1-His and TS2-His were incubated with the substrate GGPP overnight at $32{ }^{\circ} \mathrm{C}$. The reaction products were analysed by GC-MS. TS catalyses GGPP to produce a major product (taxa-4(5),11(12)-diene (1)) and a minor product (taxa-4(20),11(12)-diene (2)), while boiled TSs have no TS activity. $\mathrm{m} / \mathrm{z} 122$ is a characteristic ion of taxadienes. The taxadiene confirmed by NMR analysis was used as a reference standard (Standard). EIC, extracted ion chromatograms; OPP, pyrophosphoric acid. c, Analysis of the activity of $\mathrm{T} 5 \alpha \mathrm{H}$ and two unknown CYP725As in vitro. The in vitro enzyme assay was carried out with the purified taxadiene substrate and yeast microsomes, each including one of the six CYPs ( T5 $\alpha \mathrm{H} 1, \mathrm{~T} 5 \alpha \mathrm{H} 2, \mathrm{~T} 5 \alpha \mathrm{H} 3, \mathrm{TbT} 5 \alpha \mathrm{H}, 55326109$ or 55305455$)$ and $\mathrm{CPR}$. T5 $\alpha \mathrm{H} 1 / 2 / 3 \mathrm{can}$ produce three oxygenated taxadiene products (5(12)-oxa-3(11)-cyclotaxane (3), 5(11)-oxa-3(11)-cyclotaxane (4) and taxa-4(20),11(12)-dien-5 $\alpha$-ol (5)), whereas no catalytic compounds were observed for 55326109, 55305455 and CPR. T. brevifolia taxadiene 5- $\alpha$-hydroxylase (TbT5 $\alpha \mathrm{H}$ ), shown to have taxadiene 5 - $\alpha$-hydroxylase activity, was used as a positive control. d, Kinetic evaluation of GGPP oxidation catalysed by TS1 (blue circles) and TS2 (red rectangles). The $x$ axis indicates the substrate GGPP concentration, while the $y$ axis shows the velocity $(\mathrm{V})$ of enzymatic reaction. $K_{\mathrm{m}}=5.5 \pm 1.6 \mu \mathrm{M}(\mathrm{TS} 1), K_{\mathrm{m}}=8.6 \pm 1.5 \mu \mathrm{M}(\mathrm{TS} 2)$, $k_{\text {cat }}=1705 \mathrm{~s}^{-1}$ (TS1) and $k_{\text {cat }}=3282 \mathrm{~s}^{-1}$ (TS2). The data are shown as means $\pm \mathrm{s} . \mathrm{d}$. ( $n=3$ biologically independent samples). e, Quantitative real-time PCR analysis of the transcription levels of TS1 and TS2 in the Taxus cell line LC treated with $100 \mu \mathrm{M}$ MeJA for the indicated times. The relative gene expression levels are represented as the average fold change $\left(2^{-\Delta \Delta C t}\right)$. The Taxus actin 1 gene (7G702435613) was used as an internal reference. The data are shown as means \pm s.d. ( $n=3$ biologically independent samples). f, Biosynthesis pathway of paclitaxel in T. chinensis var. mairei. The solid arrows indicate the identified steps in the paclitaxel pathway, whereas the dashed arrows show the hypothetical steps. The compounds in the pathway are shown in black and the catalytic enzymes are shown in blue. T5 $\alpha \mathrm{H}$, taxadiene 5- $\alpha$-hydroxylase; T13 $\alpha \mathrm{H}$, taxane 13- $\alpha$-hydroxylase; TAT, taxadien-5- $\alpha$-ol O-acetyltransferase; $\mathrm{T} 10 \beta \mathrm{H}$, taxane 10- $\beta$-hydroxylase; T14 $\beta \mathrm{H}$, taxoid $14-\beta$-hydroxylase; $\mathrm{T} 2 \alpha \mathrm{H}$, taxoid 2 - $\alpha$-hydroxylase; T7 $\beta \mathrm{H}$, taxoid 7 - $\beta$-hydroxylase; TBT, 2 - $\alpha$-hydroxytaxane 2-O-benzoyltransferase; DBAT, 10-deacetylbaccatinlII 10-O-acetyltransferase; BAPT, baccatin III amino phenylpropanoyl-13-O-transferase; DBTNBT, 3'-N-debenzoyl-2'-deoxytaxol N-benzoyl transferase. 
1 lignan-terpene, 1 terpene-alkaloid and 9 putative gene clusters (Supplementary Tables 20 and 27). Two gene clusters (clusters I and II) belonging to the terpene cluster were involved in paclitaxel biosynthesis because cluster I contained the TS2, TS3, T5 $\alpha \mathrm{H} 2$ and T5 $\alpha \mathrm{H} 3$ genes, and cluster II included TS1. Except for these five genes, other related enzymes in the paclitaxel synthesis pathway were not included in any gene clusters. However, we found that most of the known genes involved in paclitaxel biosynthesis, including TAT2, DBAT, TS1/2/3, T7 $\beta \mathrm{H} 1 / 2, \mathrm{~T} 13 \alpha \mathrm{H} 1 / 2, \mathrm{~T} 10 \beta \mathrm{H} 1 / 2 / 3$, $\mathrm{T} 5 \alpha \mathrm{H} 1 / 2 / 3$ and $\mathrm{T} 14 \beta \mathrm{H}$, are located on a small $71.82-\mathrm{Mb}$ region on chromosome 9 (designated the T13 $\alpha \mathrm{H} 2-\mathrm{DBAT}$ segment: base pairs (bp) 19994572-91811351; Extended Data Fig. 6c). Therefore, many

a

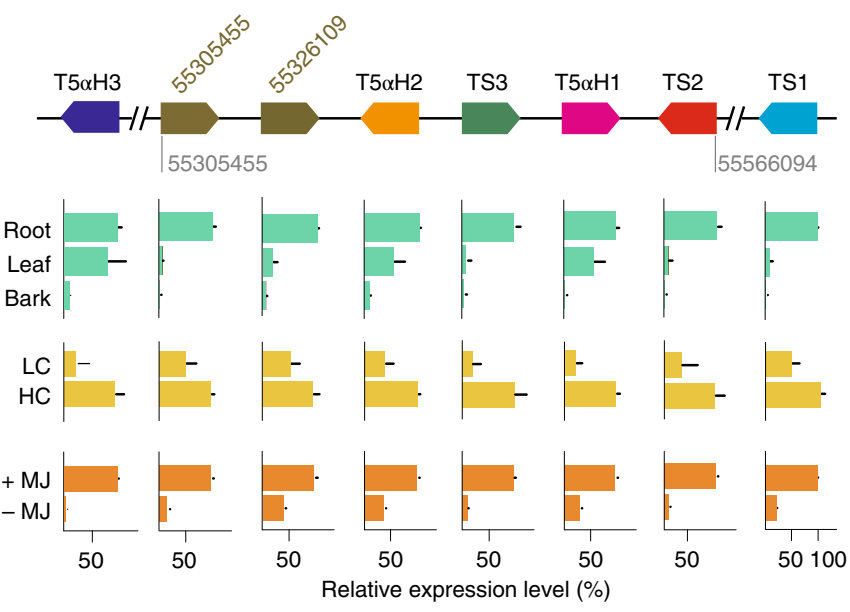

c

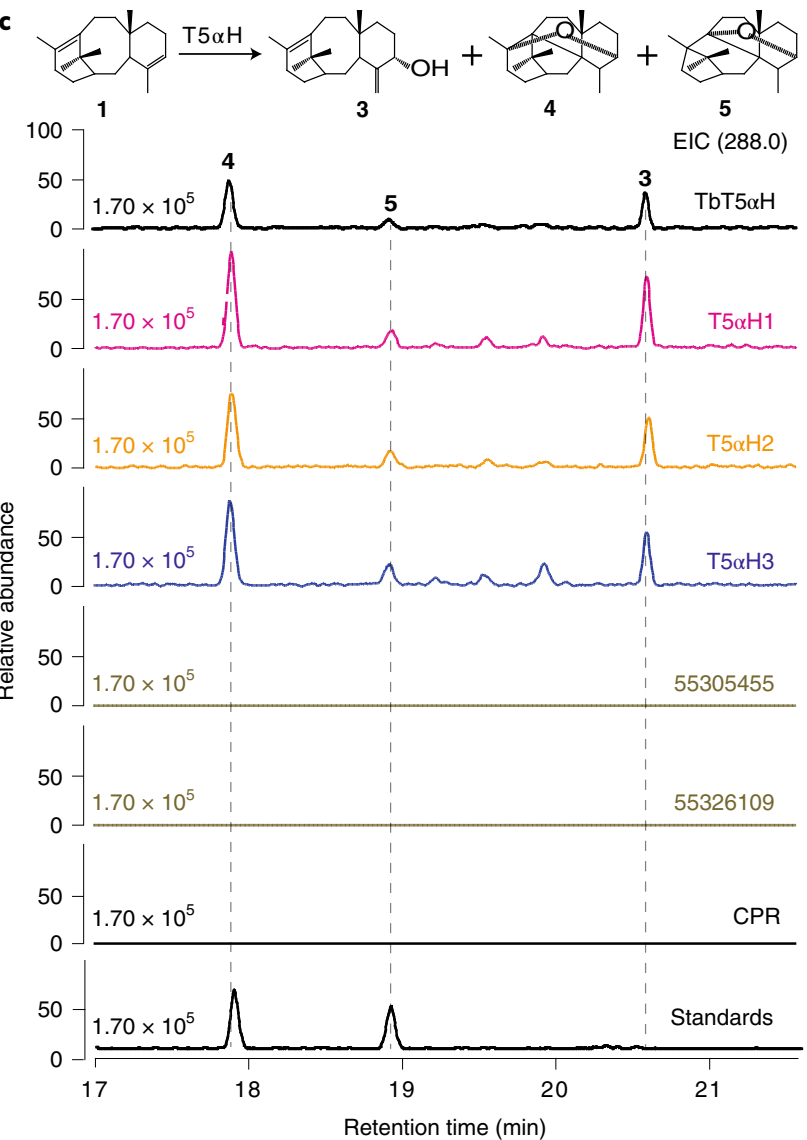

genes that play roles in different steps of the paclitaxel synthesis pathway are located in a limited genomic region, implying that there might be a coordinated regulatory mechanism of their gene expression. It would be an important future project to investigate whether the genes are organized in a larger-scale gene cluster to achieve better collaborative expression.

To date, all known TS enzymes are homologous to TS1 (amino acid homology $>90 \%$ ) (Supplementary Table 21). Our study showed that TSs could be encoded by two distinct types of TS genes resulting from gene duplication events in Taxus. As a representative of the new type of TS enzyme, TS2 only has approximately $77-78 \%$ amino acid homology with the reported TS enzymes (Supplementary Table 21)
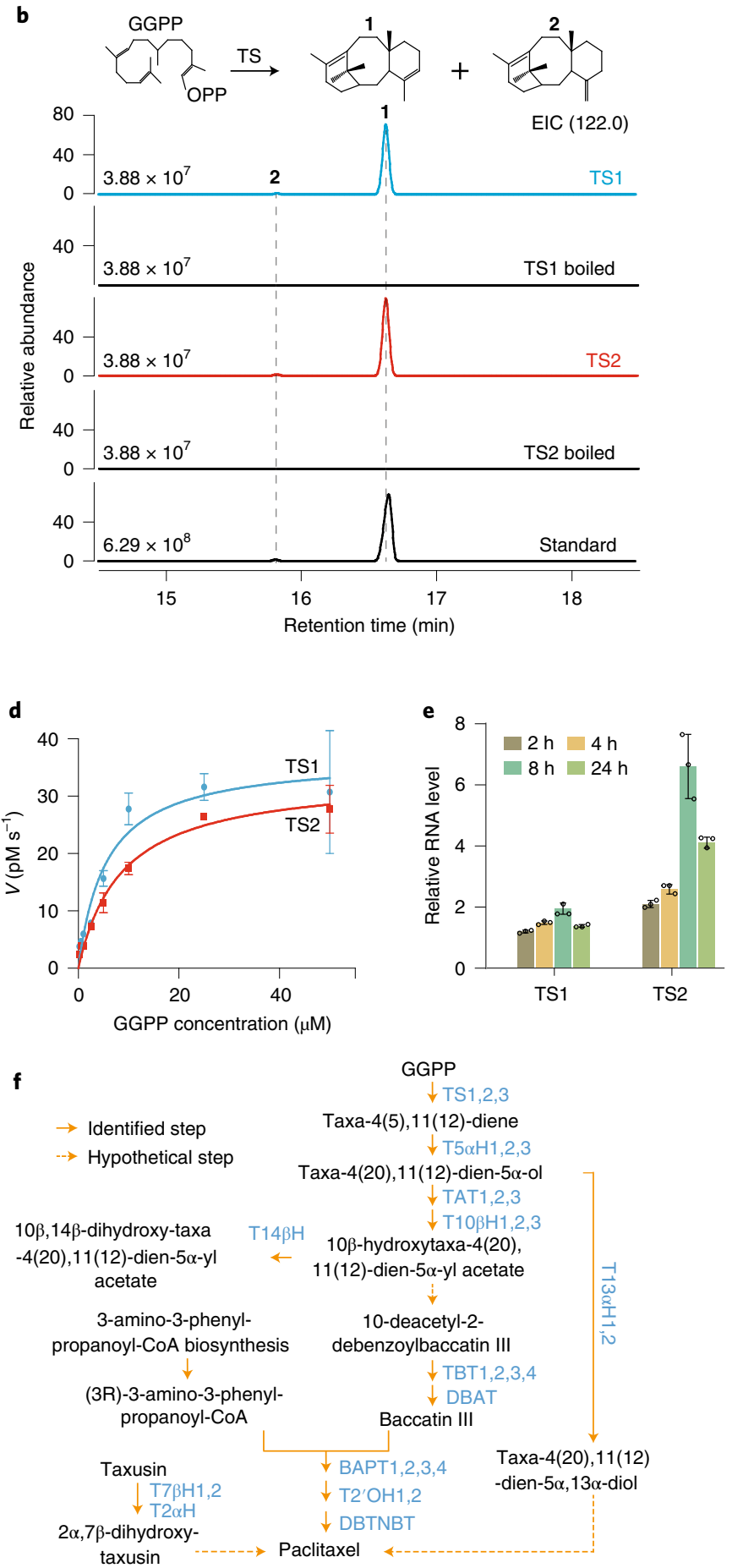
and exhibits more robust induced expression characteristics in treatment with jasmonates (Fig. 3e). The different properties of these two types of TS enzymes imply a new Taxus defence regulation mechanism. In Taxus, the excessive synthesis of taxanes is not conducive to its growth or development, although these chemicals play an essential role in defence responses. It is therefore necessary to accurately and efficiently control the taxane level in cells in response to environmental changes. Our results provide a new hypothesis to explain the regulation of taxane levels in plant cells. When there are no biotic or abiotic stresses, jasmonate signalling is blocked, and TS1 is responsible for taxane biosynthesis to maintain taxanes at a basic level. However, once insect attack or other stresses occur, jasmonate signalling is activated, and TS2 is rapidly expressed to quickly increase the taxane content in cells.

In addition, we tried to explore the application potential of TS2 in bioengineering. Bian et al. reported an engineered Escherichia coli strain with TbTS (belonging to Type I) for the taxadiene prod$\mathrm{uct}^{37}$. We replaced the TbTS gene with the TS2 gene (Extended Data Fig. 8a). After 60 hours of fermentation, we found that the taxadiene titre from the strain containing TS2 was over ten times higher than that from the strain containing TbTS, while the $\mathrm{OD}_{600}$ of the two strains was not much different (Extended Data Fig. 8b,c). This result shows the great potential of TS2 in bioengineering to produce taxadiene in the future.

We also explored the function of two unknown CYP725As (55305455 and 55326109) in the taxadiene cluster using the well-established $\mathrm{T} 5 \alpha \mathrm{H}$ reaction assay (Fig. $3 \mathrm{c}$ and Supplementary Fig. 4h). We further incubated yeast microsomes that included $55305455, \mathrm{~T} 5 \alpha \mathrm{H} 1$ and cytochrome $\mathrm{P} 450$ reductase (CPR) with taxadiene as a substrate at the same time and analysed the reaction products by gas chromatography mass spectrometry (GCMS). As shown in Supplementary Fig. 6, we detected only 5(12)-oxa-3(11)-cyclotaxane, 5(11)-oxa-3(11)-cyclotaxane and taxa-4(20),11(12)-dien-5 $\alpha$-ol, which can be obtained by catalysing taxadiene by the T $5 \alpha \mathrm{H} 1$ enzyme. The same result was obtained with 55326109 protein in the reaction system (Supplementary Fig. 6). These results suggest that the unknown CYP725As are not involved in the subsequent reaction catalysed by $\mathrm{T} 5 \alpha \mathrm{H}$. However, the tissue expression specificity of 55305455 and 55326109 was similar to that of TS and T5 $\alpha \mathrm{H}$ in the cluster, and both of them exhibited higher expression levels in roots than in leaf and bark tissues (Fig. 3a). The real-time PCR assay validated that their expression was induced by jasmonate in Taxus cells (Extended Data Fig. 6e), which is consistent with paclitaxel accumulation (Supplementary Fig. 1). Moreover, the gene-to-gene coregulation network showed that 55305455 and 55326109 were correlated with DBAT and T5 $\alpha \mathrm{H} 1$, respectively (Extended Data Fig. 6a). These results indicate that the two CYPs may play a role in paclitaxel biosynthesis and metabolism and are worthy of in-depth study in the future.

\section{Methods}

Plant materials and genome sequencing. Seeds of a single female T. chinensis var. mairei were collected from the natural range of Taxus $\left(113^{\circ} 89^{\prime} 55^{\prime \prime} \mathrm{N}\right.$, $28^{\circ} 26^{\prime} 32^{\prime \prime} \mathrm{E}$ ) in the Liuyang region, Changsha city, Hunan Province, China, in November 2015. Single embryos and endosperm were induced as calli ${ }^{23,38}$.

For sequencing of the haploid tissue, DNA was extracted from the endosperm callus of T. chinensis var. maire ${ }^{23}$. The DNA quality was checked by agarose gel electrophoresis and a Qubit fluorimeter (Thermo Fisher). The paired-end libraries with a 500-bp insert length were prepared by following the Illumina protocols. Sequencing of the library was performed on the Illumina HiSeq 2500 system. For the PacBio Sequel analysis, SMRTbell TM libraries were prepared according to the manufacturer's protocol for the sequencing platform. Four independent $\mathrm{Hi}-\mathrm{C}$ libraries were constructed and sequenced on an Illumina HiSeq 2500 (PE125 bp) at Annoroad Gene Technology Co.

For circular consensus sequencing, genomic DNA was extracted from frozen leaves using the DNeasy Plant Mini Kit (Qiagen). A 15-kilobase DNA SMRTbell library was constructed and sequenced on a PacBio Sequel II platform; these sequencing reads are known as highly accurate long reads, or HiFi reads.
Genome assembly and gene annotation. The uncorrected PacBio reads were assembled using wtdbg2 (ref. ${ }^{39}$ ), the fastest sequence assembler for long noisy reads. The assembly reached the best continuity with the following parameters: -k, 0; -p, 19; -K, 5000; -S1; -aln-noskip-tidy-reads, 5000;-edge-min, 2; rescue-low-cov-edges. The software Arrow in the GenomicConsensus package (https://github.com/PacificBiosciences/GenomicConsensus) was applied to generate the consensus sequences from the primary assembly. The raw PacBio reads were aligned to the assembly of red bean ${ }^{39}$ using pbalign (v.0.3.1) with the default parameters, and then the alignment was passed to Arrow (v.2.2.2) to produce the corrected assembly. The consensus process was performed iteratively twice. Further polishing of the assembly genome was conducted using Pilon ${ }^{40}$ with Illumina data, with the following parameters: -fix, all; -mindepth, $0.4 ;-\mathrm{K}, 65$; threads, 24 ; -minmq, 30;-minqual, 30;-changes.

For $\mathrm{Hi}-\mathrm{C}$ assembly, the clean $\mathrm{Hi}-\mathrm{C}$ sequencing data were mapped to the genome draft by HiC-Pro (v.2.7.8) ${ }^{41}$, and the library quality was assessed by counting the number of unique valid paired-end reads. Only unique valid paired-end reads were maintained for downstream analysis. We used the $\mathrm{Hi}$ - $\mathrm{C}$ data to align and correct the contigs for misassembly through the Juicer ${ }^{42}$ pipeline and the $3 \mathrm{D}$-DNA pipeline ${ }^{43}$. The assembly package Lachesis ${ }^{44}$ was applied to perform clustering, ordering and orienting on the basis of the normalized $\mathrm{Hi}-\mathrm{C}$ interactions. For each pseudochromosome group, the exact contig order and directions were obtained through a weighted directed acyclic graph. We filled the gaps among contigs in the pseudochromosomes using TGS-Gapcloser (v.1.01) ${ }^{45}$ by two rounds with continuous long-read and HiFi data $(26 \mathrm{~Gb})$, respectively. After the filling progress, we further removed the redundant contigs that were not anchored to the chromosomes using Purge Haplotigs (v.1.03) ${ }^{46}$

For assembly assessment, the RNA-seq reads of eight tissues (including female strobilus, female leaf, female bark of stem, female root, male strobili, male leaf, male bark of stem and male root) and HC and LC were mapped to assess the assembly quality. The average mapping rate of all RNA-seq datasets was subsequently calculated by software HISAT2 (ref. ${ }^{47}$ ) with the following parameter: score-min, L, 0, -0.1 .

For repeat annotation and analyses, repetitive elements in the Taxus genome were identified through a combination of de novo and homology-based approaches. De novo prediction of repeat elements was carried out using RepeatModeler (v.1.0.1, http://www.repeatmasker.org/RepeatModeler/). For homology-based annotation, the repeat element libraries from Repbase ${ }^{48}$, the Institute for Genomic Research ${ }^{49}$ and the annotated Ginkgo biloba genome were merged with the de-novo-derived library to create the whole dataset. The dataset was then used to mask identified TEs in the Taxus genome with RepeatMasker (v.4.0.5, http://www.repeatmasker.org). We identified LTRs with the LTR_retriever method $^{50}$. Specifically, LTR_finder ${ }^{50}$ and LTRharvest ${ }^{51}$ were first used to identify all the existing LTR sequences in the Taxus genome according to the basic sequence rules of LTRs. The candidate LTR RTs were filtered to remove non-LTR RT repeat elements or those with large amounts of tandem repeats or gaps. Especially in fragmented genome assemblies, these requirements hugely reduce the number of LTR RT candidates but ensure that only full-length LTR RTs are analysed. We integrated the results and discarded false positives using the LTR_retriever pipeline; we then estimated insertion times $(T)$ on the basis of $T=D / 2 \mu$, where $D$ is the divergence rate and $\mu$ is the neutral mutation rate $\left(7.34573 \times 10^{-10}\right)^{36}$

For the annotation of protein-coding genes, gene structure prediction was performed using ab initio, homology-based and RNA-seq-based pipelines. For the ab initio annotation, SNAP ${ }^{52}$, Augustus ${ }^{53}$ and GlimmerHMM were applied. Eight species (Arabidopsis thaliana ${ }^{54}$, Oryza sativa ${ }^{55}$, Gnetum montanum ${ }^{56}$, Picea abies $^{25}$, Ginkgo biloba ${ }^{35}$, Selaginella moellendorffii ${ }^{57}$, Pinus taed ${ }^{58}$ and Amborella trichopod $a^{59}$ ) were chosen for homology annotation to predict protein-coding genes using GeneWise ${ }^{60}$. To generate annotation results based on transcripts, RNA-seq alignment files were generated using TopHat2 (ref. ${ }^{61}$ ) and assembled via Cufflinks ${ }^{62}$, and the program PASA $^{63}$ was used to align spliced transcripts and annotate candidate genes. Finally, gene models predicted from three approaches were merged by $\mathrm{EVM}^{64}$. The functions of protein-coding genes were identified by mapping sequences against the Gene Ontology ${ }^{65}$, InterProScan ${ }^{66}$, Swiss-Prot (http://www.uniprot.org/) ${ }^{67}, \operatorname{TrEMBL}^{68}$ and TAIR databases ${ }^{69}$.

Identification of WGD. Genome-wide duplications were searched in the Taxus genome. Self-alignment of the assembled genome sequence was performed using metablast as described previously ${ }^{70}$. All-versus-all paralogue analysis in the Taxus genome was performed using reciprocal best hits from primary protein sequences by self-Blastp in Taxus. Reciprocal best hits are defined as reciprocal best Blastp matches with an $E$-value threshold of $10^{-5}$, a $c$-score (Blast score/best Blast score) threshold of 0.3 (ref. ${ }^{71}$ ) and an alignment length threshold of 100 amid acids. The value of $K_{\mathrm{s}}$ of reciprocal best hit gene pairs was calculated on the basis of the YN model in KaKs_Calculator v.2.0 (ref. ${ }^{72}$ ). Synteny analysis was performed on Taxus protein-coding genes using MCScanX ${ }^{73}$ to identify WGD events with the default parameters from the top ten self-Blastp hits. $K_{\mathrm{s}}$ and $4 \mathrm{DTv}$ were calculated for Taxus syntenic block gene pairs.

Genome mining for CYP450s and gene clusters. For the identification and classification of CYP450 genes, hmmsearch was used to identify CYP450 genes 
in the Taxus genome with PF00067 from the Pfam database ${ }^{74}$. The classification of the 649 CYP 450 genes was executed by alignment with the CYP450 database ${ }^{75}$ using standard sequence similarity cut-offs, with definite standards of $97 \%, 55 \%$ and $40 \%$ for allelic, subfamily and family variants, respectively. According to the standardized CYP450 nomenclature ${ }^{76}$, CYP450s were divided into A-type and non-A-type CYP450s, and phylogenetic analysis of CYP450 genes was performed for A-type and non-A-type CYP450s. Neighbour-joining phylogenetic trees were constructed using the MEGA7 package with homologous amino acid sequences ${ }^{77}$.

For genome mining for gene clusters involved in plant specialized metabolism, PlantiSMASH ${ }^{31}$ was used to search for potential gene clusters using the default parameters and the GFF (General Feature Format) annotation files of the software. Gene groups were identified by in silico analysis on the basis of the following criteria: (1) the distance between two adjacent CYP450 genes in one group should be less than $5.26 \mathrm{Mb}$, and (2) one group should contain at least seven CYP450 genes.

RNA-seq data analysis for candidate genes in the paclitaxel biosynthesis pathway. All tissues, including female strobilus, leaf, bark of stem, and root and male strobili, leaf, bark of stem, and root, were mapped to the Taxus genome, and the fragments per kilobase of transcript per million mapped reads value was calculated using HISAT2 and StringTie ${ }^{76}$. Expression data from female bark, female roots and female leaves were used to identify the genes associated with paclitaxel biosynthesis. First, we selected genes that were more highly expressed in roots or bark than in leaves. Second, the genes were further confirmed in two Taxus half-sib cell lines (HC and LC) with distinct accumulation patterns of paclitaxel, and the genes should be highly expressed in HC. The differentially expressed genes were filtered using edge $\mathrm{R}^{77}$ with $\log \mathrm{FC}>1$ and $\mathrm{FDR}<0.05$. We obtained 1,638 genes that met the above thresholds. Gene-to-gene networks were constructed using the expression matrix from MeJA-induced cell line (0, 2, 4, 8 and 24h) RNA-seq data. Pearce correlation analysis was performed with the known functional genes as the target genes. Hypothesis development for the Pearson correlation was performed, and pairs with $\mathrm{P}<0.05$ remained.

Functional characterization of TS genes. The open reading frames of TS1 (ctg6088_gene.1) and TS2 (ctg5306_gene.4) were cloned by PCR with reverse transcription from the Taxus cell line. Plant-Ploc (http://www.csbio.sjtu.edu.cn/ bioinf/plant-multi/), ChloroP (http://www.cbs.dtu.dk/services/ChloroP/) and TargetP (http://www.cbs.dtu.dk/services/TargetP/) were used for the prediction of the plastidial target sequence. The 60-residue N-terminally truncated TS1 and TS2 genes were inserted into the E. coli expression vector pET28b to form the constructs pET28b::TS1 and pET28b::TS2, respectively. All expression plasmids were constructed using the Hieff Clone One Step Cloning Kit (YEASEN), and the primers used in this work are given in Supplementary Table 28. For the in vitro enzyme assay, the enzyme assays were performed in a final volume of $500 \mu \mathrm{l}$ of buffer ( $25 \mathrm{mM}$ HEPES, pH 8.5, 10\% glycerol, $5 \mathrm{mM}$ DTT, $5 \mathrm{mM}$ sodium ascorbate, $5 \mathrm{mM}$ sodium metabisulfite and $1 \mathrm{mM} \mathrm{MgCl}_{2}$ ) containing $100 \mu \mathrm{g}$ of purified protein and $100 \mu \mathrm{M}$ GGPP (Sigma-Aldrich). The reaction mixture was overlaid with $500 \mu \mathrm{l}$ of pentane (Macklin, GC-MS grade) and incubated overnight at $32^{\circ} \mathrm{C}$. In addition, the mixture was vortexed, and the pentane overlay was subsequently removed by centrifugation at 5,000 r.p.m. for $10 \mathrm{~min}$ and concentrated by $\mathrm{N}_{2}$ gas before GC-MS analysis. Inactivated TSs-His6 was used as the control. Taxa-4(5),11(12)-diene (1) and taxa-4(20),11(12)-diene (2) preparations were performed according to a previous study with the taxadiene-producing E. coli strain T2 (harbouring pMH1, pFZ81 and $\mathrm{pXC02})^{37}$. The organic solutions containing crude compounds 1 and 2 were concentrated on ice under $\mathrm{N}_{2}$ gas and redissolved in dimethyl sulfoxide for the purification of compounds $\mathbf{1}$ and $\mathbf{2}$ by thin layer chromatography. The purity and concentration were determined by GC-MS.

For the determination of kinetic parameters, standard enzyme assays were carried out in a total volume of $100 \mu$ l containing buffer ( $25 \mathrm{mM}$ HEPES, $\mathrm{pH} 8.5$, $10 \%$ glycerol, $5 \mathrm{mM}$ DTT, $5 \mathrm{mM}$ sodium ascorbate, $5 \mathrm{mM}$ sodium metabisulfite and $1 \mathrm{mM} \mathrm{MgCl}_{2}$ ), $36 \mu \mathrm{g}$ (TS1) or $17 \mu \mathrm{g}$ (TS2) of recombinant proteins and seven different concentrations of GGPP $(0.2,0.5,1,2.5,5,10,25$ and $50 \mu \mathrm{M})$, which were spiked with $\left.{ }^{1-3} \mathrm{H}\right]$-GGPP (American Radiolabeled Chemicals, $30 \mathrm{Ci} \mathrm{mM}^{-1}$ ). Hot $\left[{ }^{1-3} \mathrm{H}\right]$-GGPP was diluted 400 times using cool GGPP (Sigma, $1 \mathrm{mg} \mathrm{ml}^{-1}$ ). The reaction mixtures were incubated at $32^{\circ} \mathrm{C}$ for $30 \mathrm{~min}$ and then quenched for $10 \mathrm{~min}$ using $100 \mu \mathrm{l}$ of stop solution (containing $1 \mathrm{M}$ EDTA and $4 \mathrm{M} \mathrm{NaOH}$ ). The reaction mixture was extracted with $800 \mu \mathrm{l}$ of $n$-hexane (vortexed for $10 \mathrm{~s}$ at 12,000 r.p.m. for $2 \mathrm{~min}$ ), and $400 \mu \mathrm{l}$ of the $n$-hexane layer was subsequently removed and mixed with $2 \mathrm{ml}$ of the liquid scintillation cocktail. The total radioactivity of the reaction products was measured using a liquid scintillation counter (Tri-Carb 2910TR, Perkin Elmer). The kinetic constant was calculated by a nonlinear regression fit to the Michaelis-Menten equation using OriginPro v.8.6 (OriginLab) ${ }^{78}$.

E. coli TS2 was constructed by replacing TbTS with TS2 on the basis of the previous taxadiene-producing E. coli TbTS (harbouring pMH1, pFZ81 and pXC02 and coexpressing nine genes-AtoB, ERG13, tHMG1, ERG12, ERG8, MVD1, IdI, GGPPS and TbTS-in E. coli) $)^{37}$ (Extended Data Fig. 8a). The E. coli strains T2 and TS2 were cultivated in $50-\mathrm{ml}$ flasks containing $30 \mathrm{ml}$ of $\mathrm{LB}$ medium at $37^{\circ} \mathrm{C}$ with $100 \mathrm{mgl}^{-1}$ ampicillin, $50 \mathrm{mgl}^{-1}$ kanamycin and $34 \mathrm{mgl}^{-1}$ chloramphenicol. When the $\mathrm{OD}_{600}$ reached approximately $0.1,1 \mathrm{mM}$ isopropyl $\beta$-D-1-thiogalactopyranoside was added to the cultures along with $3 \mathrm{ml}$ of dodecane; the bacteria were then cultivated at $28^{\circ} \mathrm{C}$. The experiments were repeated four times. For cell concentration $\left(\mathrm{OD}_{600}\right)$ and taxadiene measurement, 100- $\mu$ l cultures and 30- $\mu \mathrm{l}$ organic layers were collected at set intervals (at 8, 13, 22, 37, 46, 60, 72 and $84 \mathrm{~h}$ ). The produced taxadiene was detected with GC-MS and quantified with the nonyl acetate standard (Aladdin).

Reporting Summary. Further information on research design is available in the Nature Research Reporting Summary linked to this article.

\section{Data availability}

The T. chinensis var. mairei genome project has been deposited in the Genome Sequence Archive at the National Genomics Data Center, and is accessible at http:/ bigd.big.ac.cn/ under BioProject no. PRJCA003841. Whole-genome and RNA-seq data were deposited in the Genome Sequence Archive database under accession nos CRA004292, CRA003496 and CRA004255. The T. chinensis var. mairei genome data have also been deposited at NCBI under BioProject no. PRJNA730337 and are publicly accessible at https://www.ncbi.nlm.nih.gov/Bioproject/?term $=\mathrm{PR}$ JNA730337. Source data are provided with this paper.

\section{Code availability}

In-house Python and R scripts for gene location, P450 analysis and heat-map analyses can be freely downloaded at GitHub (https://github.com/liaoqinggang/ Taxus_genome_pipelines).

Received: 26 November 2020; Accepted: 10 June 2021; Published online: 15 July 2021

\section{References}

1. Christenhusz, M. et al. A new classification and linear sequence of extant gymnosperms. Phytotaxa 19, 55-70 (2010).

2. Hao, D. C., Xiao, P. G., Huang, B., Ge, G. B. \& Yang, L. Interspecific relationships and origins of Taxaceae and Cephalotaxaceae revealed by partitioned Bayesian analyses of chloroplast and nuclear DNA sequences. Plant Syst. Evol. 276, 89-104 (2008).

3. Wani, M. C., Taylor, H. L., Wall, M. E., Coggon, P. \& McPhail, A. T. Plant antitumor agents. VI. Isolation and structure of Taxol, a novel antileukemic and antitumor agent from Taxus brevifolia. J. Am. Chem. Soc. 93, 2325-2327 (1971).

4. Sabzehzari, M., Zeinali, M. \& Naghavi, M. R. Alternative sources and metabolic engineering of Taxol: advances and future perspectives. Biotechnol. Adv. 43, 107569 (2020).

5. Nicolaou, K. C. et al. Total synthesis of Taxol. Nature 367, 630-634 (1994).

6. Baloglu, E. \& Kingston, D. G. I. A new semisynthesis of paclitaxel from baccatin III. J. Nat. Prod. 62, 1068-1071 (1999).

7. Fett-Neto, A. G., DiCosmo, F., Reynolds, W. F. \& Sakata, K. Cell culture of Taxus as a source of the antineoplastic drug Taxol and related taxanes. Nat. Biotechnol. 10, 1572-1575 (1992).

8. Kumar, P. et al. Hyper-production of Taxol from Aspergillus fumigatus, an endophytic fungus isolated from Taxus sp. of the Northern Himalayan region. Biotechnol. Rep. (Amst.) 24, e00395 (2019).

9. Ajikumar, P. K. et al. Isoprenoid pathway optimization for Taxol precursor overproduction in Escherichia coli. Science 330, 70-74 (2010).

10. Kuang, X., Sun, S., Wei, J., Li, Y. \& Sun, C. Iso-seq analysis of the Taxus cuspidata transcriptome reveals the complexity of Taxol biosynthesis. BMC Plant Biol. 19, 210 (2019).

11. Croteau, R., Ketchum, R. E. B., Long, R. M., Kaspera, R. \& Wildung, M. R. Taxol biosynthesis and molecular genetics. Phytochem. Rev. 5, 75-97 (2006).

12. Wildung, M. R. \& Croteau, R. A cDNA clone for taxadiene synthase, the diterpene cyclase that catalyzes the committed step of Taxol biosynthesis. J. Biol. Chem. 271, 9201-9204 (1996).

13. Howat, S. et al. Paclitaxel: biosynthesis, production and future prospects. N. Biotechnol. 31, 242-245 (2014).

14. Sanchez-Muñoz, R. et al. A novel hydroxylation step in the taxane biosynthetic pathway: a new approach to paclitaxel production by synthetic biology. Front. Bioeng. Biotech. https://doi.org/10.3389/fbioe.2020.00410 (2020).

15. Walker, K. \& Croteau, R. Taxol biosynthesis: molecular cloning of a benzoyl-CoA:taxane $2 \alpha$-O-benzoyltransferase cDNA from Taxus and functional expression in Escherichia coli. Proc. Natl Acad. Sci. USA 97, 13591-13596 (2000)

16. Fett-Neto, A. G., Melanson, S. J., Sakata, K. \& DiCosmo, F. Improved growth and Taxol yield in developing calli of Taxus cuspidata by medium composition modification. Nat. Biotechnol. 11, 731-734 (1993).

17. Wasternack, C. Action of jasmonates in plant stress responses and development-applied aspects. Biotechnol. Adv. 32, 31-39 (2014).

18. Cusido, R. M. et al. A rational approach to improving the biotechnological production of taxanes in plant cell cultures of Taxus spp. Biotechnol. Adv. 32, 1157-1167 (2014) 
19. Zhang, M. et al. Transcriptome-wide identification and screening of WRKY factors involved in the regulation of Taxol biosynthesis in Taxus chinensis. Sci. Rep. 8, 5197 (2018).

20. Lenka, S. K. et al. Jasmonate-responsive expression of paclitaxel biosynthesis genes in Taxus cuspidata cultured cells is negatively regulated by the bHLH transcription factors TcJAMYC1, TcJAMYC2, and TcJAMYC4. Front. Plant Sci. 6, 115 (2015).

21. Yu, C. et al. Tissue-specific study across the stem of Taxus media identifies a phloem-specific TmMYB3 involved in the transcriptional regulation of paclitaxel biosynthesis. Plant J. 10, tjp14710 (2020).

22. Zonneveld, B. J. M. Conifer genome sizes of 172 species, covering 64 of 67 genera, range from 8 to 72 picogram. Nord. J. Bot. 30, 490-502 (2012).

23. Li, Y. et al. A protocol of homozygous haploid callus induction from endosperm of Taxus chinensis Rehd. var. mairei. SpringerPlus 5, 659 (2016).

24. Jiao, Y. et al. Ancestral polyploidy in seed plants and angiosperms. Nature 473, 97-100 (2011).

25. Nystedt, B. et al. The Norway spruce genome sequence and conifer genome evolution. Nature 497, 579-584 (2013).

26. Zhang, Q. J. \& Gao, L. Z. Rapid and recent evolution of LTR retrotransposons drives rice genome evolution during the speciation of AA-genome Oryza species. G3 7, 1875-1885 (2017)

27. Guerra-Bubb, J., Croteau, R. \& Williams, R. M. The early stages of Taxol biosynthesis: an interim report on the synthesis and identification of early pathway metabolites. Nat. Prod. Rep. 29, 683-696 (2012).

28. Gesell, A. et al. The gymnosperm cytochrome P450 CYP750B1 catalyzes stereospecific monoterpene hydroxylation of $(+)$-sabinene in thujone biosynthesis in western redcedar. Plant Physiol. 168, 94-106 (2015).

29. Nelson, D. \& Werck-Reichhart, D. A P450-centric view of plant evolution. Plant J. 66, 194-211 (2011).

30. Wasternack, C. \& Strnad, M. Jasmonates are signals in the biosynthesis of secondary metabolites - pathways, transcription factors and applied aspects-a brief review. N. Biotechnol. https://doi.org/10.1016/j. nbt.2017.09.007 (2019).

31. Kautsar, S., Suarez, H., Blin, K., Osbourn, A. \& Medema, M. PlantiSMASH: automated identification, annotation and expression analysis of plant biosynthetic gene clusters. Nucleic Acids Res. https://doi.org/10.1093/nar/ gkx305 (2017).

32. Shang, Y. et al. Biosynthesis, regulation, and domestication of bitterness in cucumber. Science 346, 1084-1088 (2014).

33. Ben, F. \& Anne, E. O. Metabolic diversification-independent assembly of operon-like gene clusters in different plants. Science 320, 543-547 (2008).

34. Bertioli, D. et al. The genome sequence of Arachis duranensis and Arachis ipaensis, the diploid ancestors of cultivated peanut. Nat. Genet. https://doi. org/10.1038/ng.3517 (2016).

35. Guan, R. et al. Draft genome of the living fossil Ginkgo biloba. Gigascience 5, 49 (2016).

36. De La Torre, A., Li, Z., Van de Peer, Y. \& Ingvarsson, P. Contrasting rates of molecular evolution and patterns of selection among gymnosperms and flowering plants. Mol. Biol. Evol. https://doi.org/10.1093/molbev/msx069 (2017).

37. Bian, G. et al. Production of taxadiene by engineering of mevalonate pathway in Escherichia coli and endophytic fungus Alternaria alternata TPF6. Biotechnol. J. https://doi.org/10.1002/biot.201600697 (2017).

38. Li, Y. et al. Induction of half-sib embryonic callus and production of taxiod compounds from Taxus chinensis var. mairei. Int. J. Agric. Biol. 21, 719-725 (2019).

39. Ruan, J. \& Li, H. Fast and accurate long-read assembly with wtdbg2. Nat. Methods 17, 155-158 (2020).

40. Wang, J. et al. Pilon: an integrated tool for comprehensive microbial variant detection and genome assembly improvement. PLOS ONE 9, e112963 (2014).

41. Servant, N. et al. HiC-Pro: an optimized and flexible pipeline for $\mathrm{Hi}-\mathrm{C}$ data processing. Genome Biol. 16, 259 (2015).

42. Durand, N. et al. Juicer provides a one-click system for analyzing loop-resolution Hi-C experiments. Cell Syst. 3, 95-98 (2016).

43. Dudchenko, O. et al. De novo assembly of the Aedes aegypti genome using Hi-C yields chromosome-length scaffolds. Science 356, 92-95 (2017).

44. Burton, J. N. et al. Chromosome-scale scaffolding of de novo genome assemblies based on chromatin interactions. Nat. Biotechnol. 31, 1119-1125 (2013).

45. $\mathrm{Xu}, \mathrm{M}$. et al. TGS-GapCloser: a fast and accurate gap closer for large genomes with low coverage of error-prone long reads. GigaScience https://doi. org/10.1093/gigascience/giaa094 (2020).

46. Roach, M. J., Schmidt, S. A. \& Borneman, A. R. Purge Haplotigs: allelic contig reassignment for third-gen diploid genome assemblies. BMC Bioinform. 19, 460 (2018).

47. Kim, D., Langmead, B. \& Salzberg, S. L. HISAT: a fast spliced aligner with low memory requirements. Nat. Methods 12, 357-360 (2015).

48. Bao, W., Kojima, K. K. \& Kohany, O. Repbase update, a database of repetitive elements in eukaryotic genomes. Mob. DNA 6, 11 (2015).
49. Ouyang, S. \& Buell, C. R. The TIGR Plant Repeat Databases: a collective resource for the identification of repetitive sequences in plants. Nucleic Acids Res. 32, D360-D363 (2004).

50. Ou, S. \& Jiang, N. LTR_retriever: a highly accurate and sensitive program for identification of long terminal repeat retrotransposons. Plant Physiol. 176, 1410-1422 (2018).

51. Ellinghaus, D., Kurtz, S. \& Willhoeft, U. LTRharvest, an efficient and flexible software for de novo detection of LTR retrotransposons. BMC Bioinform. 9, 18 (2008).

52. Korf, I. Gene finding in novel genomes. BMC Bioinform. 5, 59 (2004).

53. Stanke, M. \& Waack, S. Gene prediction with a hidden Markov model and a new intron submodel. Bioinformatics 19, ii215-ii225 (2003).

54. The Arabidopsis Genome Initiative. Analysis of the genome sequence of the flowering plant Arabidopsis thaliana. Nature 408, 796-815 (2000).

55. Goff, S. A. et al. A draft sequence of the rice genome (Oryza sativa L. ssp. japonica). Science 296, 92-100 (2002).

56. Wan, T. et al. A genome for gnetophytes and early evolution of seed plants. Nat. Plants 4, 82-89 (2018).

57. Banks, J. A. et al. The Selaginella genome identifies genetic changes associated with the evolution of vascular plants. Science 332, 960-963 (2011).

58. Zimin, A. et al. Sequencing and assembly of the 22-Gb loblolly pine genome. Genetics 196, 875-890 (2014).

59. Amborella Genome Project. The Amborella genome and the evolution of flowering plants. Science 342, 1241089 (2013).

60. Birney, E., Clamp, M. \& Durbin, R. GeneWise and Genomewise. Genome Res 14, 988-995 (2004).

61. Kim, D. et al. TopHat2: accurate alignment of transcriptomes in the presence of insertions, deletions and gene fusions. Genome Biol. 14, R36 (2013).

62. Trapnell, C. et al. Differential gene and transcript expression analysis of RNA-seq experiments with TopHat and Cufflinks. Nat. Protoc. 7 , 562-578 (2012).

63. Haas, B. J. Improving the Arabidopsis genome annotation using maximal transcript alignment assemblies. Nucleic Acids Res. 31, 5654-5666 (2003).

64. Haas, B. J. et al. Automated eukaryotic gene structure annotation using EVidenceModeler and the Program to assemble spliced alignments. Genome Biol. 9, R7 (2008)

65. Blake, J. A., Chan, J., Kishore, R., Sternberg, P. W. \& Li, Y. Gene Ontology Consortium: going forward. Nucleic Acids Res. 43, 1049-1056 (2015).

66. Mitchell, A. L. et al. InterPro in 2019: improving coverage, classification and access to protein sequence annotations. Nucleic Acids Res. 47, 351-360 (2019).

67. Bairoch, A. \& Apweiler, R. The SWISS-PROT protein sequence data bank and its new supplement TREMBL. Nucleic Acids Res. 24, 21-25 (1996).

68. Stoesser, G., Sterk, P., Tuli, M. A., Stoehr, P. J. \& Cameron, G. N. The EMBL nucleotide sequence database. Nucleic Acids Res. 25, 7-13 (1997).

69. Lamesch, P. et al. The Arabidopsis Information Resource (TAIR): improved gene annotation and new tools. Nucleic Acids Res. 40, 1202-1210 (2012).

70. Bailey, J. A., Church, D. M., Ventura, M., Rocchi, M. \& Eichler, E. E. Analysis of segmental duplications and genome assembly in the mouse. Genome Res. 14, 789-801 (2004).

71. Putnam, N. H. et al. The amphioxus genome and the evolution of the chordate karyotype. Nature 453, 1064-1071 (2008).

72. Wang, D. P., Wan, H. L., Zhang, S. \& Yu, J. $\gamma$-MYN: a new algorithm for estimating $\mathrm{Ka}$ and $\mathrm{Ks}$ with consideration of variable substitution rates. Biol. Direct 4, 20 (2009).

73. Wang, Y. et al. MCScanX: a toolkit for detection and evolutionary analysis of gene synteny and collinearity. Nucleic Acids Res. 40, 49-63 (2012).

74. Eddy, S. R. Accelerated profile HMM searches. PLoS Comput. Biol. 7, e1002195 (2011).

75. Nelson, D. R. The cytochrome p450 homepage. Hum. Genomics 4 , 59-65 (2009).

76. Durst, F. \& Nelson, D. R. Diversity and evolution of plant P450 and P450-reductases. Drug Metab. Drug Interact. 12, 189-206 (1995).

77. Kumar, S., Stecher, G. \& Tamura, K. MEGA7: Molecular Evolutionary Genetics Analysis version 0 for bigger datasets. Mol. Biol. Evol. 33, 1870-1874 (2016)

78. Grant, F. Origin Pro 8.6. Scientific Computing World (2011).

\section{Acknowledgements}

We thank T. Liu (Wuhan University) for sharing plasmids that were used for the preparation of taxadiene in E. coli. We thank Y. Zhang (Shanghai University) and S. Cheng (Agricultural Genomics Institute at Shenzhen) for helpful discussion. We thank T. Feng (Wuhan Botanical Garden, Chinese Academy of Sciences) for valuable help with TS evolution. This work was supported by the National Key R\&D Program of China (grant nos 2018YFA0903200, 2018YFA0901800 and 2020YFA0907900), Research Funds for Central Nonprofit Scientific Institution (grant no. Y2020XK23), the Elite Young Scientists Program of CAAS, the Agricultural Science and Technology Innovation Program, National Science and Technology Basic Special Project (grant no. 2017FY100100), the Scientific Research Fund of Hunan Provincial Education Department (grant no. 2016XYX001), Double First-Class Construction Project of Hunan Agricultural University (grant no. SYL201802026), Fund of the Education Department 
of Hunan Province (grant no. 18B124) and the National Natural Science Foundation of China (grant no. 32000236).

\section{Author contributions}

The ideas for the paper were conceived by J.Y., S.H. and X.X. Q.L., G.B., Q.Z., X.W., T.S. Z.Z. and Y.W. performed the bioinformatic analysis. Y.L., X.X., C.L., D.-K.R., L.G. and P.L. prepared the Taxus sequencing samples and the Taxus cell lines. J.G., H.L., D.K.-R., Y.S. and R.D. undertook the biochemistry experiments and mass spectrometry. J.Y., S.H., J.G., Q.L., Y.L. and X.X. interpreted the data and wrote the paper.

\section{Competing interests}

The authors declare no competing interests.

\section{Additional information}

Extended data is available for this paper at https://doi.org/10.1038/s41477-021-00963-5. Supplementary information The online version contains supplementary material available at https://doi.org/10.1038/s41477-021-00963-5.
Correspondence and requests for materials should be addressed to S.H. or J.Y.

Peer review information Nature Plants thanks Jing-Ke Weng, Xiaoquan Qi, Kexuan Tang and Cathie Martin for their contribution to the peer review of this work.

Reprints and permissions information is available at www.nature.com/reprints.

Publisher's note Springer Nature remains neutral with regard to jurisdictional claims in published maps and institutional affiliations.

Open Access This article is licensed under a Creative Commons

Attribution 4.0 International License, which permits use, sharing, adaptation, distribution and reproduction in any medium or format, as long as you give appropriate credit to the original author(s) and the source, provide a link to the Creative Commons license, and indicate if changes were made. The images or other third party material in this article are included in the article's Creative Commons license, unless indicated otherwise in a credit line to the material. If material is not included in the article's Creative Commons license and your intended use is not permitted by statutory regulation or exceeds the permitted use, you will need to obtain permission directly from the copyright holder. To view a copy of this license, visit http://creativecommons. org/licenses/by/4.0/.

(c) The Author(s) 202 
a

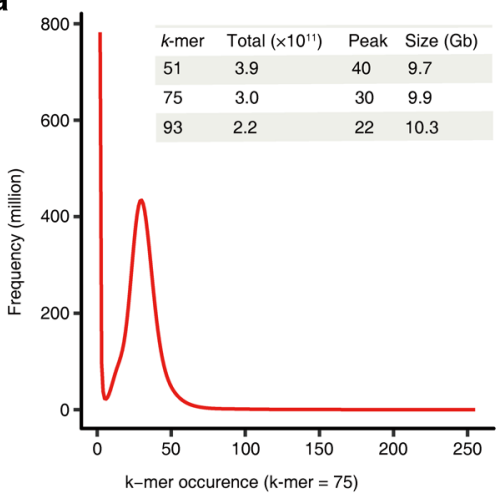

b

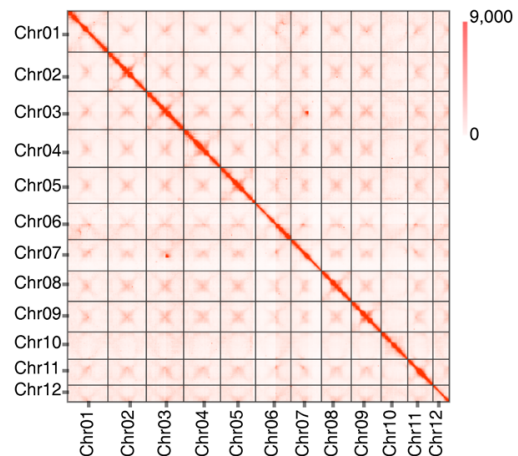

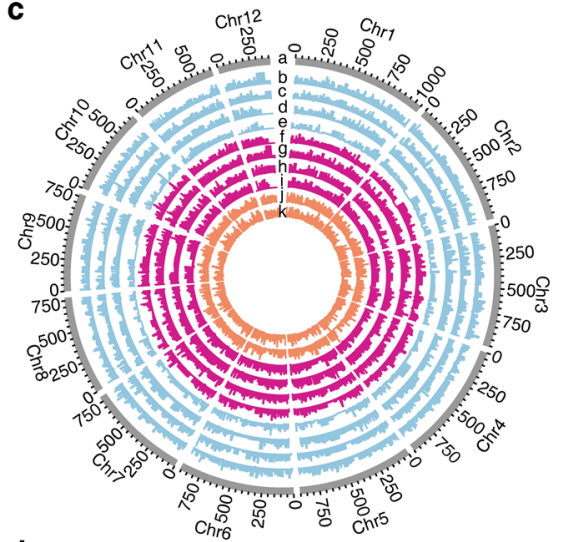

d

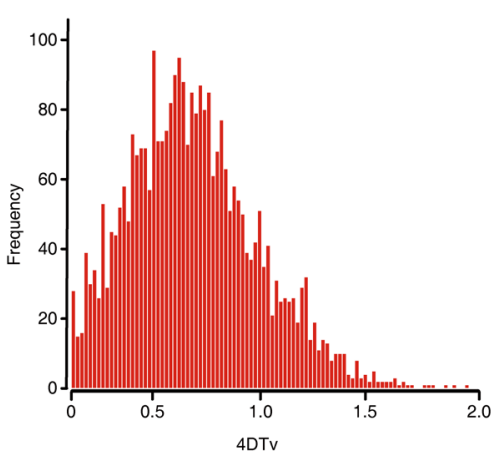

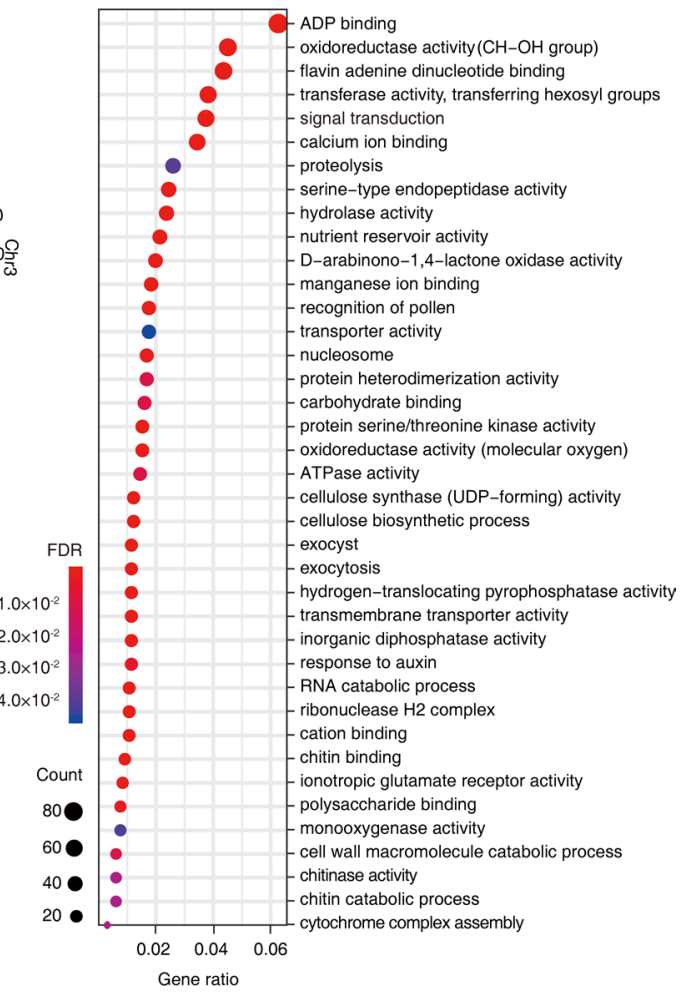

Extended Data Fig. 1 | The Taxus genomic features to complement Fig. 1. a, Genome size estimation of T. chinensis var. mairei based on k-mer distribution. The $\mathrm{X}$-axis represents the occurrence of $k$-mers, and the $\mathrm{Y}$-axis represents the frequency. The $k$-mer values for different genome sizes are shown in the inner table. b, Genome-wide all-by-all $\mathrm{Hi}-\mathrm{C}$ interaction. The heat map shows $\mathrm{Hi}-\mathrm{C}$ interactions under a resolution of $2 \mathrm{Mb}$. Darker red pixels indicate higher contact probabilities. The number on the scale bar indicates the number of links after logarithmic analysis. c, Genomic landscape of the twelve pseudochromosomes. Track a represents the length of the pseudochromosomes (Mb); b, c, d, and e show the expression of tissue-specific genes in the bark of stem, root, strobili and leaf from the male Taxus plant, respectively; $f, g, h$, and i show the expression of tissue-specific genes in the bark of stem, root, strobilus and leaf from the female Taxus plant, respectively; j and k display high- and low- producing paclitaxel cell lines, respectively. $d$, Whole genome duplication (WGD) analysis based on the substitution rate distribution of paralogs. The 4DTv values of paralogs were calculated using KaKs_ calculator with the YN model. The X-axis is the value of fourfold synonymous third-codon transversions (4DTv) for paralogous pairs in the Taxus genome, and the $\mathrm{Y}$-axis represents the frequency. e, Gene Ontology (GO) enrichment for gene families with significant expansion. GO enrichment analysis of a subset of 142 gene families with significant expansion $(p<0.05)$; FDRs were adjusted for multiple testing. The size and color of dots indicate the number of genes and false discovery rate (FDR), respectively. The $\mathrm{X}$-axis represents the gene ratio, and the $\mathrm{GO}$ terms are listed on the $\mathrm{Y}$ axis. 

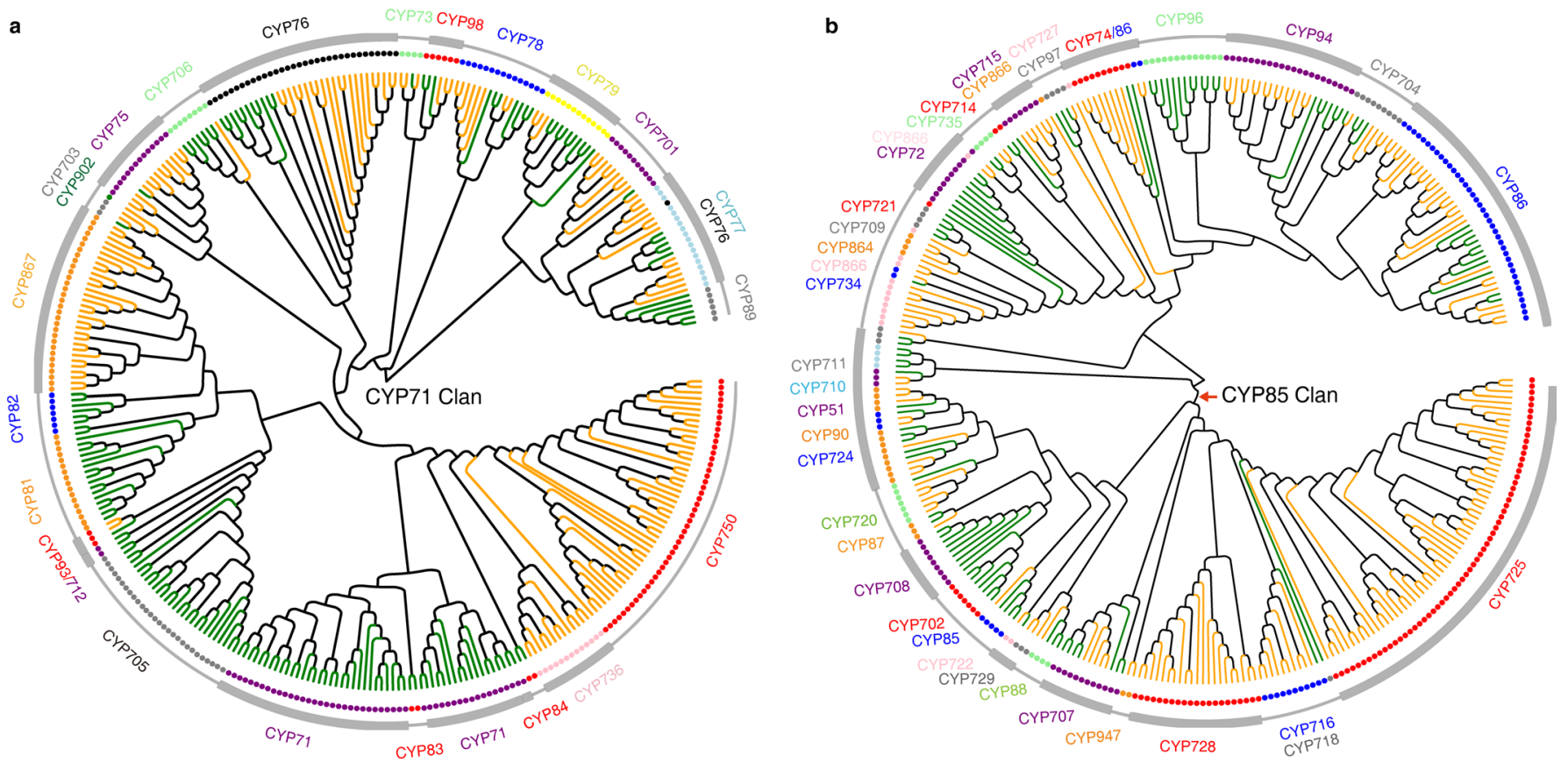

Extended Data Fig. 2 | Phylogenic analysis of A-type and non-A-type CYP450 families. a, Phylogenic analysis of A-type CYP450 families. The green and orange branches indicate the sequences from Arabidopsis and T. chinensis var. mairei, respectively. The dots represent CYP450 genes. The outermost circle indicates the CYP450 gene family. b, Phylogenic analysis of non-A-type CYP450 families. The green and orange branches indicate the sequences from Arabidopsis and T. chinensis var. mairei, respectively. The dots represent CYP450 genes. The outermost circle indicates the CYP450 gene family. 


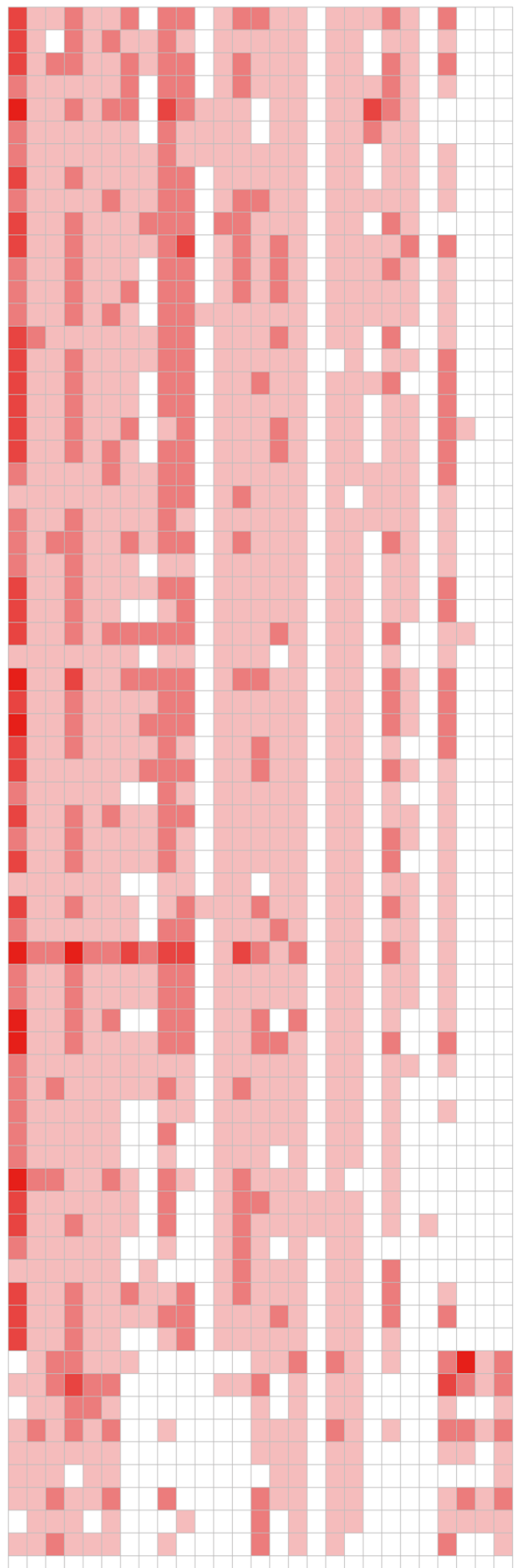

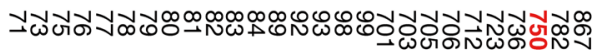

71

A type of CYP450 b

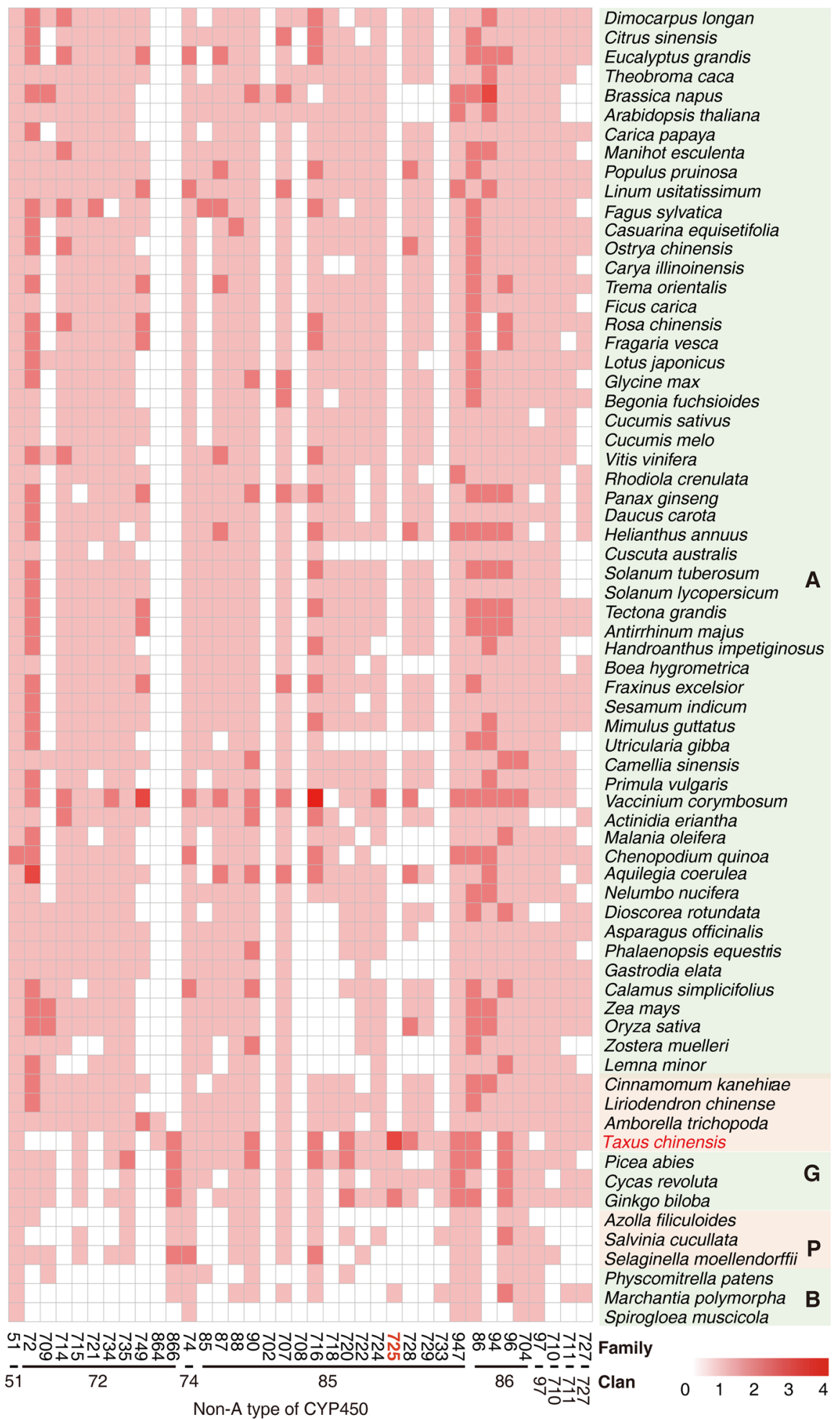

Extended Data Fig. 3 | Heat map of the number of CYP450 genes in 69 representative plant species. Each CYP450 gene family of A-type (a) and non-A-type (b) is represented as a square, with the red color representing the number of genes in the corresponding family. The depth of the red color is divided into five levels, namely, $0,1,2,3$, and 4, which correspond to 0, 1-10, 10-50, 50-100, and more than 100 genes, respectively. The family or clan name of CYP450 genes is marked below the heat map. A, Angiosperms; G, Gymnosperms; P, Pteridophytes; and B, Bryophytes. 

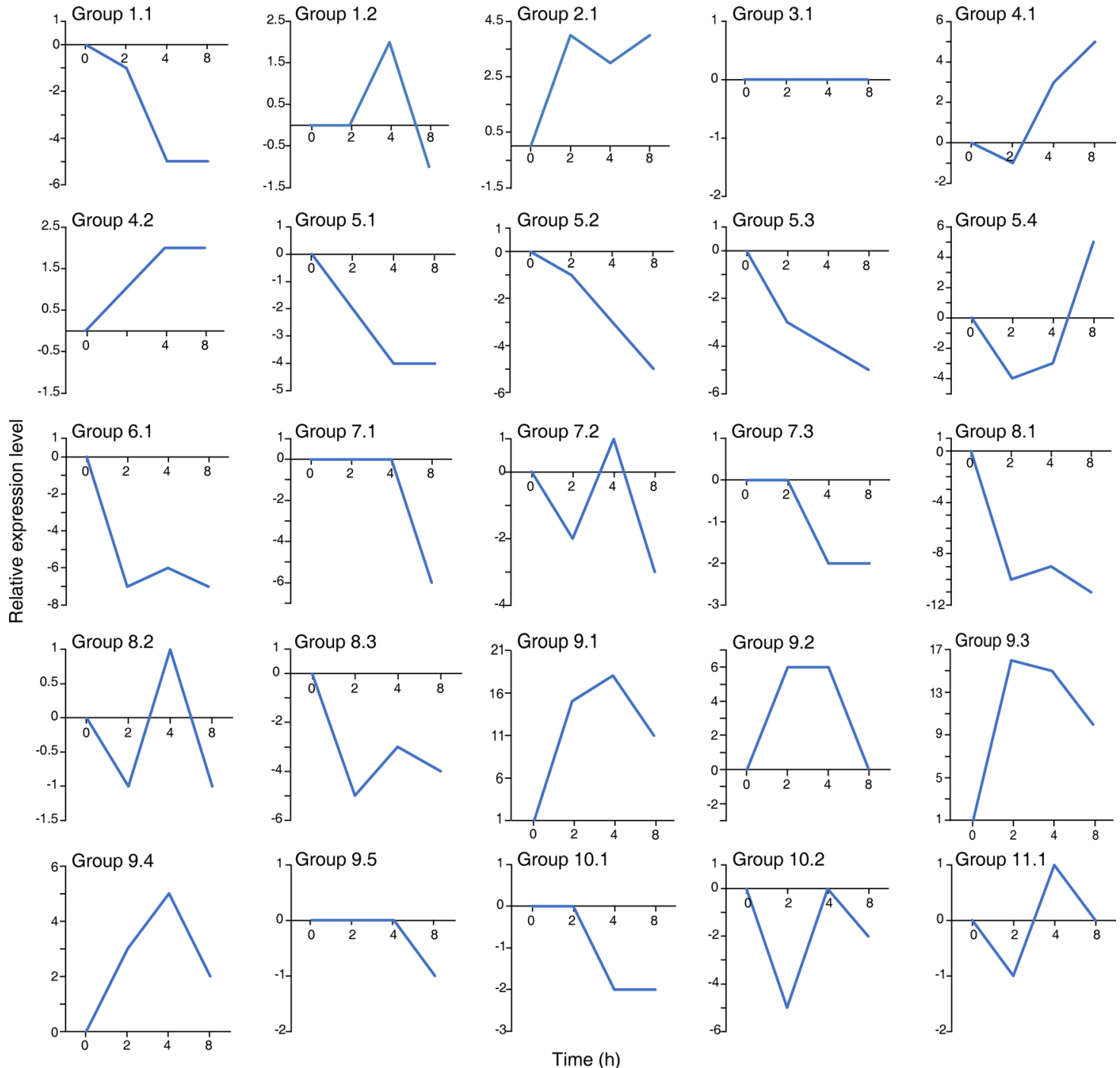

Extended Data Fig. 4 | Gene expression in response to MeJA treatment in the Taxus cell line. Group-based gene expression profiles in response to MeJA treatment. RNA sequencing analysis was performed with the Taxus cell line treated with $100 \mu \mathrm{M} \mathrm{MeJA}$ or $0.5 \%$ EtOH solution for $0,2,4$, and $8 \mathrm{~h}$. The expression of the gene group was calculated by summing the expression levels of each CYP450. Each upregulated and downregulated CYP450 was calculated as 1 and -1 , respectively, based on their FPKM (Fragments Per Kilobase of transcript per Million mapped reads) values. 


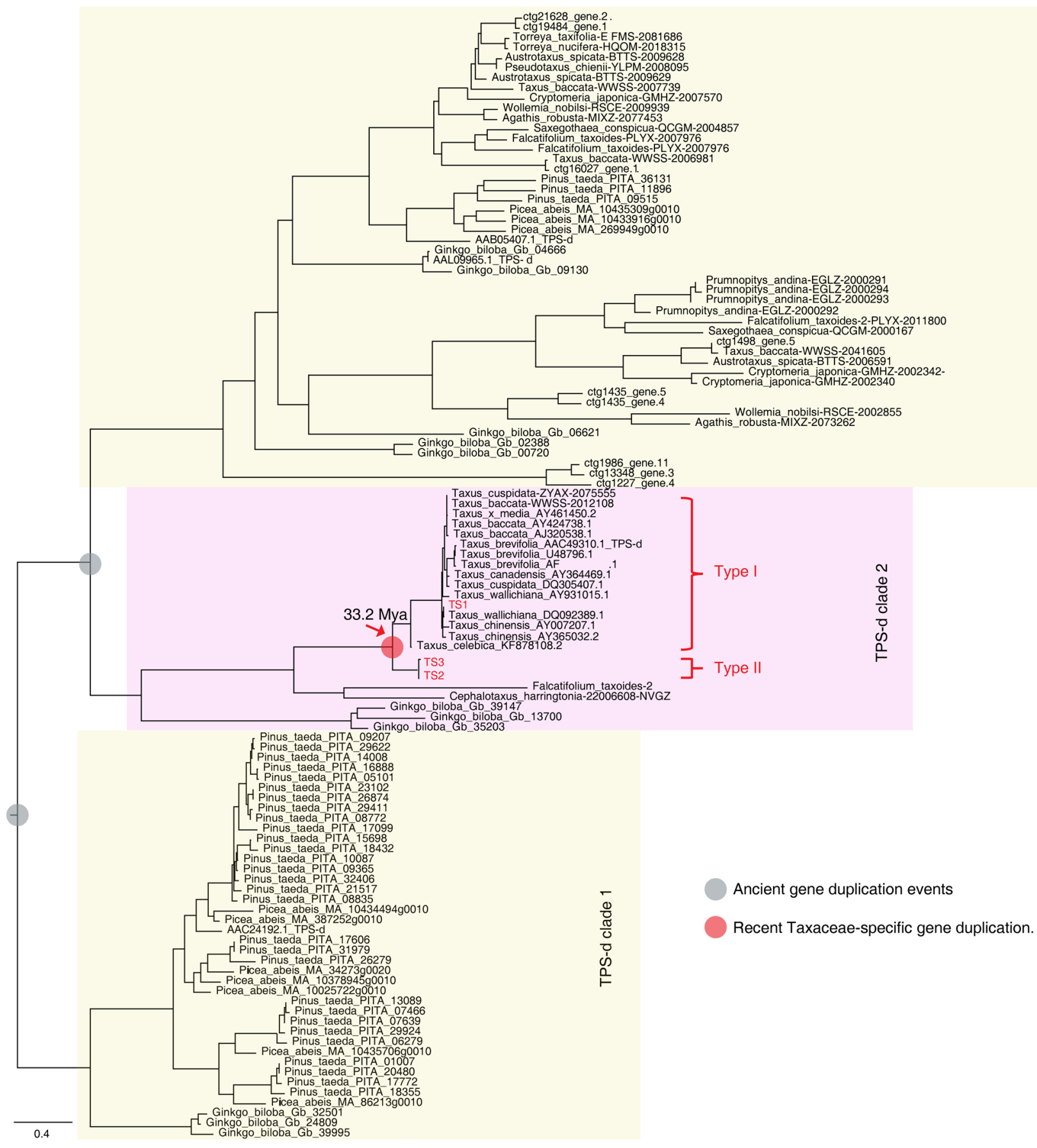

Extended Data Fig. 5 | Phylogenetic analysis of trehalose-6-phosphate synthase d subfamily (TPS-d) genes from different plants. The tree is generated from amino acid sequences by the maximum-likelihood method with 100 bootstraps. Ancient gene duplication events are indicated as gray dots, while the more recent Taxaceae-specific gene duplication is shown as a red dot. The TS1/2/3 genes in T. chinensis var. mairei are highlighted in red. 
a

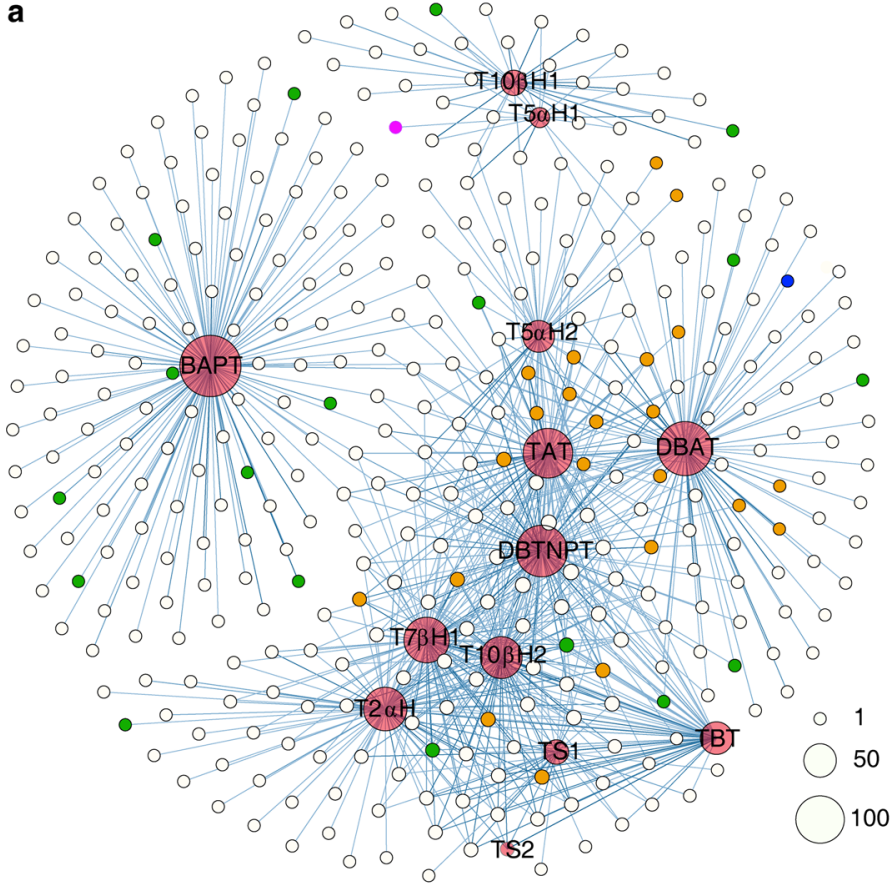

d
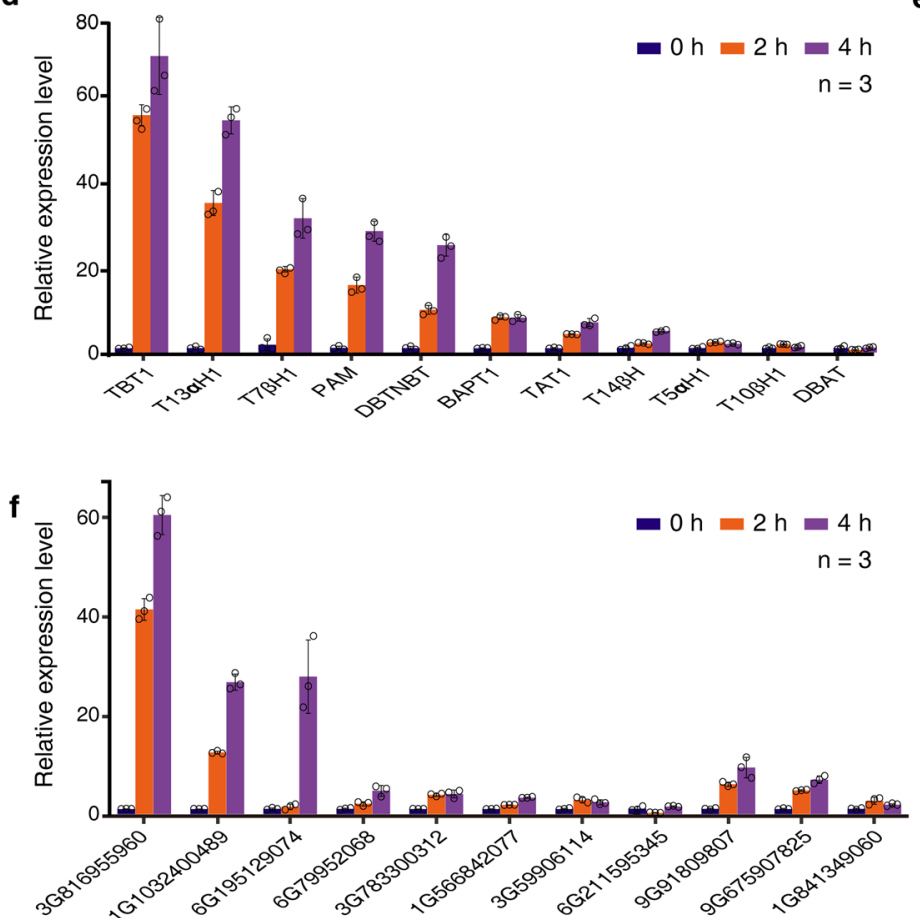

b

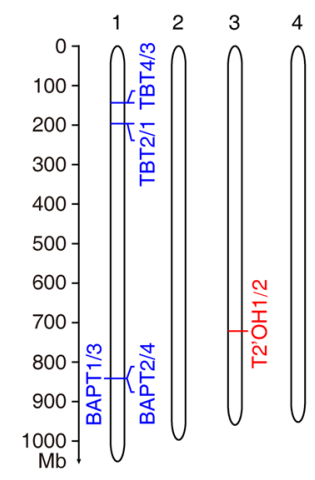

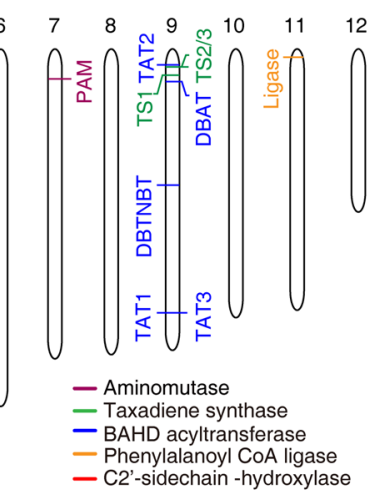

C

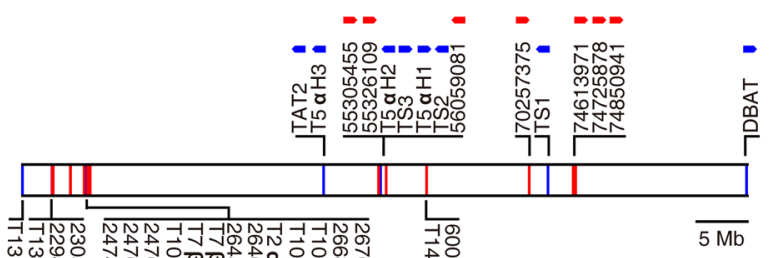

$\omega$

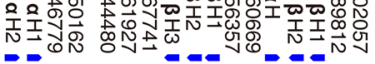

-

I Known genes | CYP725A

e

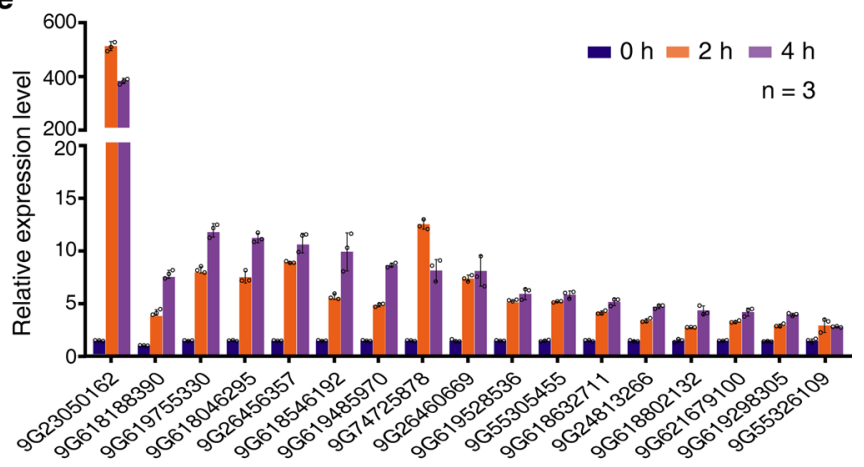

Extended Data Fig. 6 | The characteristic of gene expression and location related to the paclitaxel biosynthesis in T. chinensis var. mairei. a,

Co-expression net of paclitaxel biosynthesis genes. The genes with a Pearson correlation coefficient value above 0.75 are displayed on the net. The known paclitaxel biosynthesis genes, CYP725s, CYP450s, and the remaining genes are represented as red, orange, green, and white dots, respectively. The purple and blue dots show the two novel CYP725A genes, 55305455 and 55326109 , respectively. The size of the dot correlates with the gene number. b, Genomic location of the annotated genes known to be involved in paclitaxel biosynthesis, except for CYP450s. The different colors of the short lines indicate the different types of annotated genes and their homologs in the paclitaxel pathway; the short purple, green, blue, orange, and red lines correspond to aminomutase, taxadiene synthase, BAHD acyltransferase, ligase, and C2'-sidechain-hydroxylase. c, Defined genes and 18 novel CYP725As on chromosome 9. The known genes in the paclitaxel biosynthesis pathway (known genes) are marked by blue lines, while the unknown CYP725As are shown in red lines. The arrows show gene orientations. $\mathbf{d}-\mathbf{f}$, The relative transcript abundance of the eleven defined paclitaxel biosynthetic genes (d), the sixteen CYP725A candidates (e), and the eight TFs and three BAHD acyltransferase genes (f) in MeJA-induced Taxus cell lines by quantitative real-time PCR (qPCR) analysis. The relative gene expression levels are represented as the average fold change ( $\left.2^{-\Delta \Delta C t}\right)$. The Taxus actin 1 gene (7G702435613) was used as an internal reference. Error bars indicate standard errors from three independent biological replicates. 

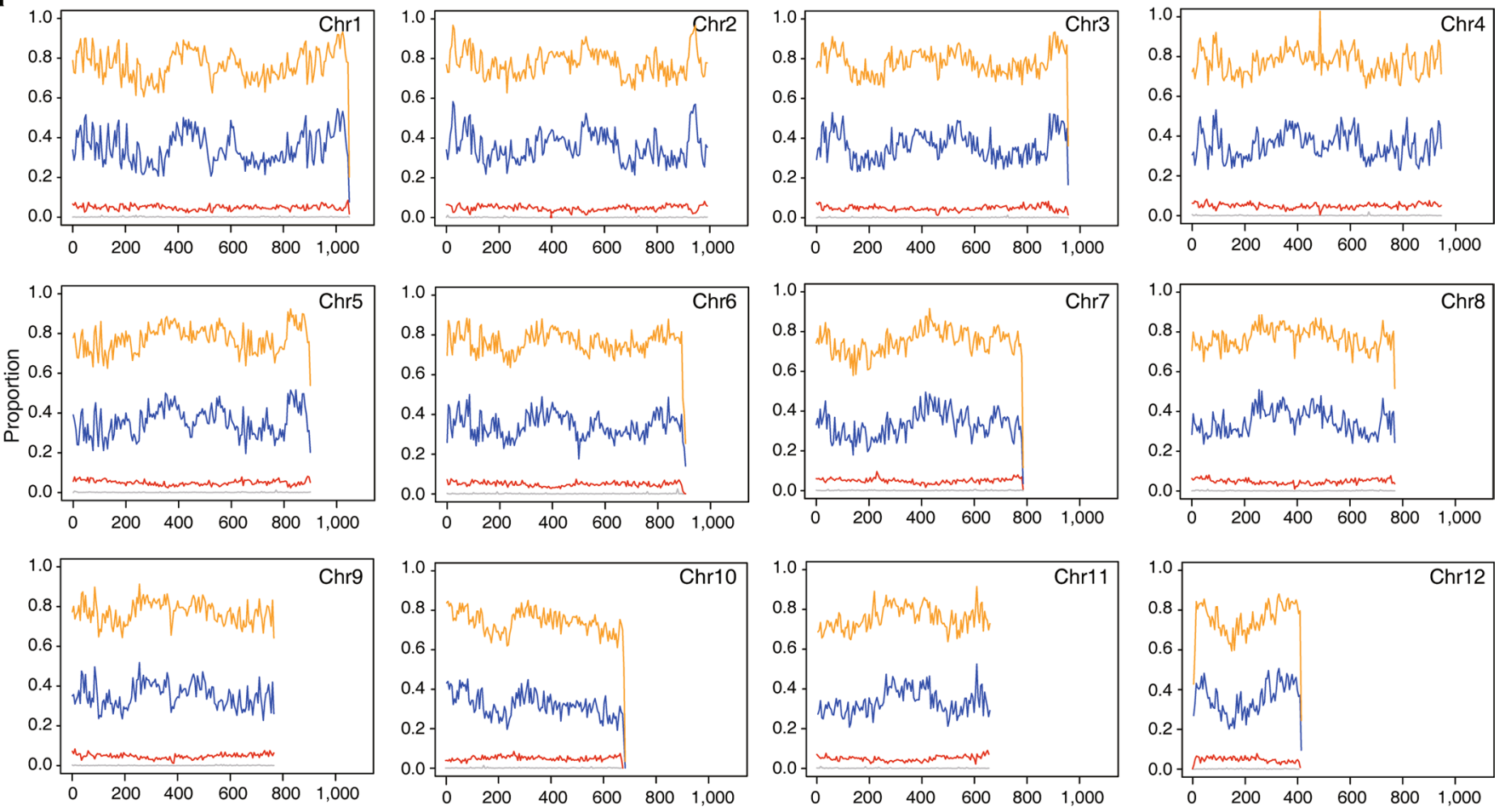

Length of chromosome $(\mathrm{Mb})$
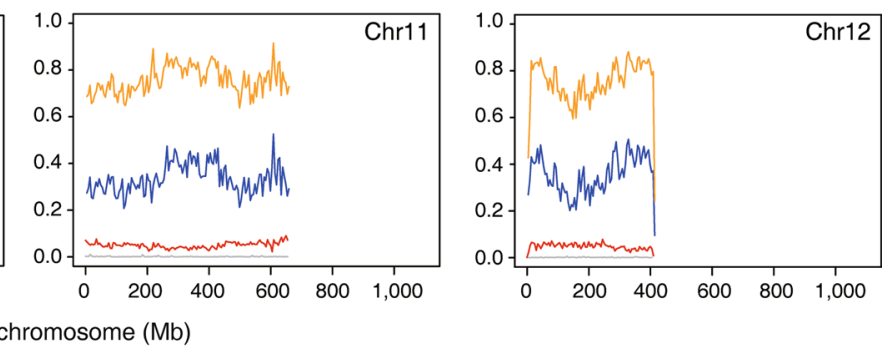

b

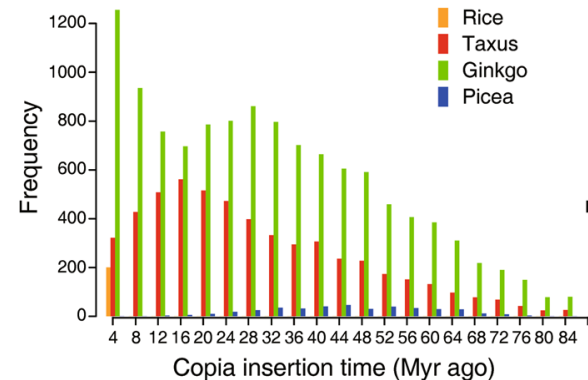

C

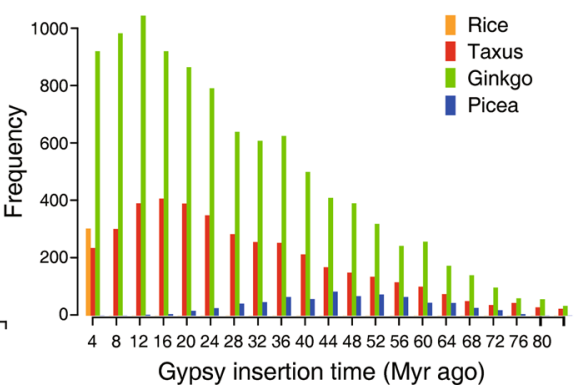

\section{d}

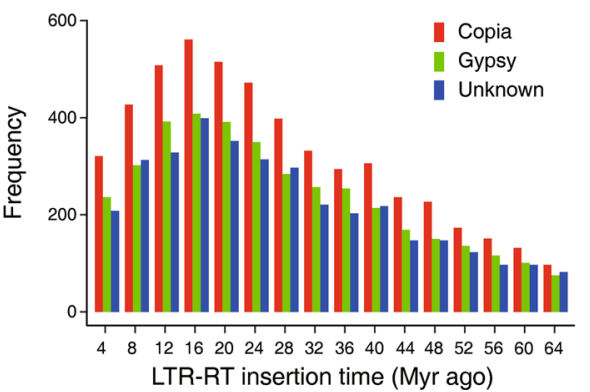

Extended Data Fig. 7 | The LTR features related to Fig. 1. a, Distribution of repeats and LTR on the chromosomes. The lines indicate different elements (Orange: repeats; Blue: Gypsy; Red, Copia; Grey: Unknown LTR). Each point on the line represents the proportion of the component in the $5 \mathrm{Mb}$ window. $\mathbf{b}$ and c, Comparison of distributions of LTR insertion times in different species. The histogram shows the distributions of insertion times calculated for Copia (b) and Gypsy (c) in Taxus, ginkgo, picea, and rice. The different colors of the columns represent the Copia and Gypsy insertions of the four plants. d,

Comparison of insertion-time distributions of different LTR elements in the Taxus. The histogram shows the distributions of the insertion times calculated for the Taxus LTR elements (Gypsy, Copia, and an unknown type). 
a

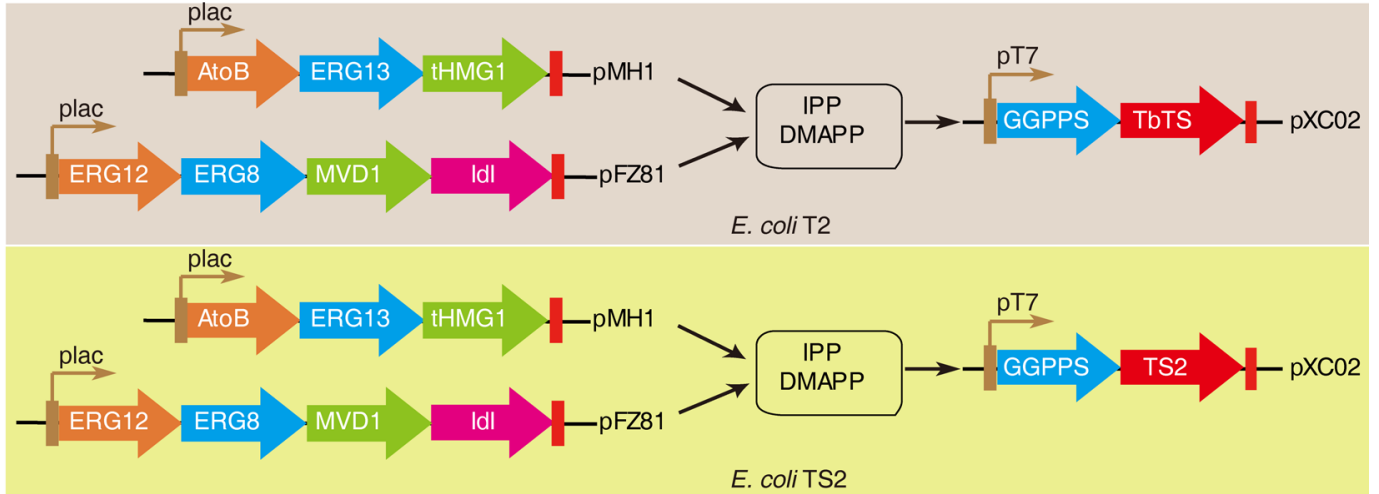

b

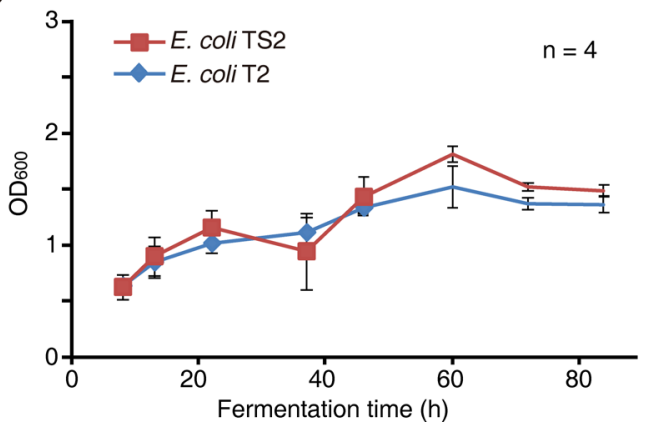

C

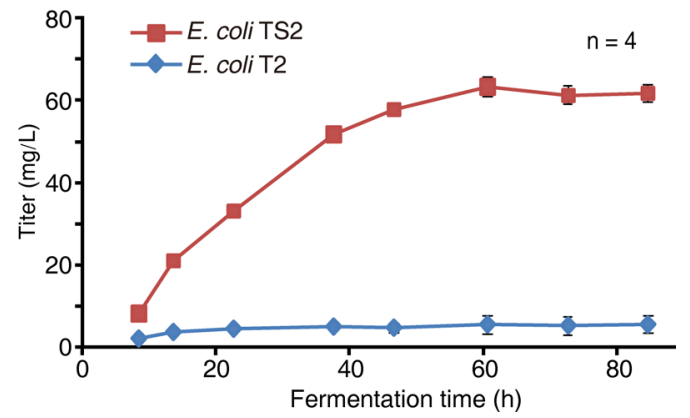

Extended Data Fig. 8 | Comparison of the two types of TS on the production of taxadiene in E. coli. a, Taxadiene-producing E. coli T2 (harboring pMH1, pFZ81, and pXC02) was constructed by coexpressing nine genes (AtoB, ERG13, tHMG1, ERG12, ERG8, MVD1, Idl, GGPPS, and TbTS) in E. coli ${ }^{29}$, while E. coli TS2 was generated by replacing TbTS with TS2 in E. coli T2; $\mathbf{b}$, The cell concentrations of the strains E. coli T2 and TS2 were measured by OD 600 at set intervals (at 8, 13, 22, 37, 46, 60, 72 and 84 hours); c, The titers of taxadiene produced by E. coli T2 and TS2 in shaking flasks. TbTS, a T. brevifolia taxadiene synthase that shares $98.42 \%$ amino acid sequence identity with TS1, represents type I TSs, while TS2, sharing $77 \%$ protein sequence identity with TS1, represents type II TSs. Error bars show standard error ( $n=4$ independent biological replicates). 


\section{Reporting Summary}

Nature Research wishes to improve the reproducibility of the work that we publish. This form provides structure for consistency and transparency in reporting. For further information on Nature Research policies, see our Editorial Policies and the Editorial Policy Checklist.

\section{Statistics}

For all statistical analyses, confirm that the following items are present in the figure legend, table legend, main text, or Methods section.

n/a Confirmed

$\bigotimes$ The exact sample size $(n)$ for each experimental group/condition, given as a discrete number and unit of measurement

\ A statement on whether measurements were taken from distinct samples or whether the same sample was measured repeatedly

The statistical test(s) used AND whether they are one- or two-sided

Only common tests should be described solely by name; describe more complex techniques in the Methods section.

Х $\square$ A description of all covariates tested

Х $\square$ A description of any assumptions or corrections, such as tests of normality and adjustment for multiple comparisons

A full description of the statistical parameters including central tendency (e.g. means) or other basic estimates (e.g. regression coefficient)

AND variation (e.g. standard deviation) or associated estimates of uncertainty (e.g. confidence intervals)

For null hypothesis testing, the test statistic (e.g. $F, t, r$ ) with confidence intervals, effect sizes, degrees of freedom and $P$ value noted Give $P$ values as exact values whenever suitable.

Х $\square$ For Bayesian analysis, information on the choice of priors and Markov chain Monte Carlo settings

Х $\square$ For hierarchical and complex designs, identification of the appropriate level for tests and full reporting of outcomes

$\triangle \square$ Estimates of effect sizes (e.g. Cohen's d, Pearson's $r$ ), indicating how they were calculated

\section{Our web collection on statistics for biologists contains articles on many of the points above.}

\section{Software and code}

Policy information about availability of computer code

Data collection DNA-seq raw NGS data was generated by sequencing using the Illumina Hiseq 2500 platform. DNA-seq raw pacbio data were generated by sequencing using the Pacbio sequel II.

RNA-seq raw data were generated by sequencing using the Illumina Hiseq2500 platform.

Data analysis All softwares used in the present study are publicly available and the corresponding software versions were described in detail in the Methods.

1.fastp v0.20.1 https://github.com/OpenGene/fastp

2.wtdbg2 v2.5 https://github.com/ruanjue/wtdbg2

3.GenomicConsensus v2.3.2 https://github.com/PacificBiosciences/GenomicConsensus

4.pilon v1.23 https://github.com/broadinstitute/pilon

5. Hi-C pro v2.11.1 https://github.com/nservant/HiC-Pro

6.juicer v1.6 https://github.com/aidenlab/juicer

7.3D-DNA v180922 https://github.com/aidenlab/3d-dna

8.TGS-Gapcloser v1.01 https://github.com/BGI-Qingdao/TGS-GapCloser

9.Purge Haplotigs v1.03 https://bitbucket.org/mroachawri/purge_haplotigs/src/master/

10.HISAT2 v2.1.0 http://daehwankimlab.github.io/hisat2/download/

11.StringTie v1.3.5 https://ccb.jhu.edu/software/stringtie/

12.RepeatModeler v1.0.1 http://www.repeatmasker.org/RepeatModeler/

13.RepeatMasker v4.0.5 http://repeatmasker.org/

14.LTR_finder v1.07 https://github.com/xzhub/LTR_Finder

15. genometools v1.5.10 http://genometools.org/pub/binary_distributions/

16.LTR_retriever v2.7 https://github.com/oushujun/LTR_retriever

17.SNAP v2013-02-16 https://github.com/KorfLab/SNAP

18.Augustus v3.2.2 https://github.com/Gaius-Augustus/Augustus 
19.GlimmerHMM v3.0.4 http://ccb.jhu.edu/software/glimmerhmm/

20.Genewise v2.2.0 https://www.ebi.ac.uk/seqdb/confluence/display/THD/GeneWise

21.Tophat2 v.2.1.1 http://ccb.jhu.edu/software/tophat/index.shtml

22.Cufflinks v2.2.1 http://cole-trapnell-lab.github.io/cufflinks/

23.PASA v2.4.1 http://pasapipeline.github.io/

24.EVM v1.1.1 https://evidencemodeler.github.io/

25.ncbi-blast v2.2.26 ftp://ftp.ncbi.nlm.nih.gov/blast/executables/blast+/LATEST/

26.MCScanX $v$ https://github.com/wyp1125/MCScanx

27.KaKs_Calculator v2.0 https://sourceforge.net/projects/kakscalculator2/

28. MEGA7 v7.0.26 https://www.megasoftware.net/dload_mac_beta

29. Plantismash v3.0.5 http://plantismash.secondarymetabolites.org/

30. edgeR R package v3.24.3 https://bioconductor.org/packages/release/bioc/html/edgeR.html

31. Jellyfish v2.1.3 https://github.com/gmarcais/Jellyfish

32. Sniffles v1.0.12 https://github.com/fritzsedlazeck/Sniffles/

33. MUSCLE v3.8.31 https://www.ebi.ac.uk/Tools/msa/muscle/

34. EMBOSS v6.6.0 http://emboss.sourceforge.net/

35. RAxML v8.2.9 https://cme.h-its.org/exelixis//web/software/raxml/index.html

36. Gblock v0.91b http://molevol.cmima.csic.es/castresana/Gblocks/Gblocks_documentation.html

37. orthoMCL v2.0.9 https://legacy.orthomcl.org/common/downloads/

38. r8s v1.81 https://sourceforge.net/projects/r8s/

39. cafe v3.0 https://github.com/hahnlab/CAFE

40. clusterProfiler R package v3.10.1 http://www.bioconductor.org/packages/release/bioc/html/clusterProfiler.html

41. pheatmap R package v1.0.12 https://www.rdocumentation.org/packages/pheatmap/versions/1.0.12

42. ggplot2 R package v3.3.3 https://cran.microsoft.com/web/packages/ggplot2/index.html

43. Gephi v0.92 https://gephi.org/

44. Pfam http://pfam.xfam.org/family/PF00067

45. Cytochrome P450 homepage http://drnelson.uthsc.edu/cytochromeP450.html

46. KAAS https://www.genome.jp/tools/kaas/

47. BUSCO v4.14 https://busco.ezlab.org/

48. Gene Ontology http://geneontology.org/

49. InterProscan v5.21-60.0 http://www.ebi.ac.uk/interpro/download/

50. Swiss-port https://www.uniprot.org/downloads

51. TrEMBL https://www.uniprot.org/downloads

52. TAIR https://www.arabidopsis.org/download/index-auto.jsp?dir=/download_files/Proteins

For manuscripts utilizing custom algorithms or software that are central to the research but not yet described in published literature, software must be made available to editors and

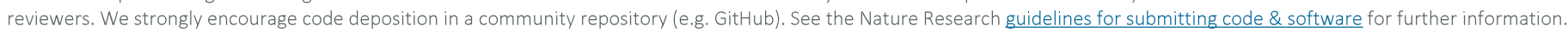

Policy information about availability of data

All manuscripts must include a data availability statement. This statement should provide the following information, where applicable:

- Accession codes, unique identifiers, or web links for publicly available datasets

- A list of figures that have associated raw data

- A description of any restrictions on data availability

The raw data used in this study have been deposited in the Genome Sequence Archive at the BIG Data Center, Beijing Institute of Genomics (BIG), Chinese Academy of Sciences and are accessible at http://bigd.big.ac.cn/gsa under bioproject PRJCA003841. The T. chinensis var. mairei genome sequences have been deposited at NCBI, under BioProject number PRJNA730337 and are publicly accessible at https://www.ncbi.nlm.nih.gov/Bioproject/?term=

PRJNA730337.

\section{Field-specific reporting}

Please select the one below that is the best fit for your research. If you are not sure, read the appropriate sections before making your selection.

$\triangle$ Life sciences

Behavioural \& social sciences

Ecological, evolutionary \& environmental sciences

For a reference copy of the document with all sections, see nature.com/documents/nr-reporting-summary-flat.pdf

\section{Life sciences study design}

All studies must disclose on these points even when the disclosure is negative.

Sample size

Sample size was measured in a way that we are able to obtain statistically differences from at least three biological replicates. For RNA-seq eight tissues from the female plant and the male plant and three half-sib cell lines were collected individually, at least three independent biological replicates were analyzed for each tissue. For MeJA treatment, 20-30 plates of Taxus cell lines were used for each assay.

Data exclusions

We excluded Taxus cell lines that displayed growth defects prior to treatment.

Replication

For measurements of RNA-seq, qRT-PCR, taxane metabolite, kinetic assay, and TS in vivo assay at least three independent measurements were statistically analyzed. 


\section{Reporting for specific materials, systems and methods}

We require information from authors about some types of materials, experimental systems and methods used in many studies. Here, indicate whether each material, system or method listed is relevant to your study. If you are not sure if a list item applies to your research, read the appropriate section before selecting a response.

\begin{tabular}{l|l} 
Materials \& experimental syste \\
\hline$n / a$ & Involved in the study \\
\hline & $\bigotimes$ Antibodies \\
$\square$ & $\square$ Eukaryotic cell lines \\
$\square$ & $\square$ Animals and other organisms \\
$\square$ & $\square$ Clinical data \\
$\square$ & $\square$ Dual use research of concern
\end{tabular}

\begin{tabular}{l|l}
\multicolumn{2}{l}{ Methods } \\
\hline n/a & Involved in the study \\
$\square$ & $\square$ ChIP-seq \\
$\searrow$ & $\square$ Flow cytometry \\
$\searrow$ & $\square$ MRI-based neuroimaging
\end{tabular}

Antibodies

Antibodies used

Flag Tag, Beyotime, China, Cat\#AF5051, Flag Tag Mouse Monoclonal Antibody, 1:1000 dilution in vitro;

$\lg (\mathrm{H}+\mathrm{L})$, Beyotime, China, Cat\#A0216, Sheep Polyclonal Antibody Conjugated with Horseradish Peroxidase, 1:1000 dilution in vitro.

Validation

The mouse monoclonal antibody for the indicated epitope tags and sheep polyclonal antibody conjugated with horseradish peroxidase (Beyotime, Cat\#AF5051 and A0216) were validated by the respective company (Beyotime: https://www.beyotime.com/ index.htm). These antibodies are commonly used in molecular biology research.

\section{Eukaryotic cell lines}

Policy information about cell lines

Cell line source(s)

Authentication

Mycoplasma contamination

Commonly misidentified lines

(See ICLAC register)
The homozygous haploid callus and two half-sib cell lines (HC, a high paclitaxel-yielding cell line; LC, a low paclitaxel-yielding line) were induced from endosperm of Taxus chinensis var. mairei by ourself.

These Taxus cell lines are published in plant science-related studies (doi:10.1186/s40064-016-2320-4, doi:10.17957) IJAB/15.0949).

All Taxus cell lines were tested regularly for Mycoplasma contamination.

No commonly misidentified cell lines were used. 